\title{
Scaling range of velocity and passive scalar spectra in grid turbulence
}

\author{
S. K. Lee, ${ }^{1}$ A. Benaissa, ${ }^{2}$ L. Djenidi, ${ }^{1}$ P. Lavoie ${ }^{3}$ and R. A. Antonia ${ }^{1}$ \\ ${ }^{1}$ School of Engineering, University of Newcastle, NSW 2308, Australia \\ ${ }^{2}$ Faculty of Engineering, Royal Military College of Canada, Ontario K7K 7B4, Canada \\ ${ }^{3}$ Institute for Aerospace Studies, University of Toronto, Ontario M3H 5T6, Canada
}

(Received 12 December 2011; accepted 28 May 2012; published online 2 July 2012; corrected 5 July 2012)

\begin{abstract}
Isotropic velocity and scalar fluctuations are closely approximated by slightly stretching a heated grid flow through a short (1.36:1) contraction. The heating is such that temperature serves as a passive scalar, and the velocity/scalar time scale ratio is about one. At small values of Taylor microscale Reynolds number $\left(10<R_{\lambda}<10^{2}\right)$, the spectrum of the temperature fluctuations has a more discernible scaling range than the spectrum of the velocity fluctuations. The scaling-range exponent for the thermal spectrum, $m_{\theta}$, exhibits a power-law function of $R_{\lambda}$ and tends to the Kolmogorov value of 5/3 more rapidly than that for the velocity spectrum, $m_{u}$. Both $m_{\theta}$ and $m_{u}$ are closer to the Kolmogorov value with the contraction than with no contraction. The trends for the present measurements supplemented with previously published data for larger $R_{\lambda}$ $\left(>10^{2}\right)$ indicate that, to obtain a $5 / 3$ scaling range, $R_{\lambda}$ must exceed $10^{3}$. The ratio $(5 / 3$ $\left.+m_{u}\right) / m_{\theta}$ is approximately 2 , in close conformity with the proposal of Danaila and Antonia ["Spectrum of a passive scalar in moderate Reynolds number homogeneous isotropic turbulence," Phys. Fluids 21, 111702 (2009)]. 2012 American Institute of Physics. [http://dx.doi.org/10.1063/1.4731295]
\end{abstract}

\section{INTRODUCTION}

The Kolmogorov" 1,2 or "K41" theory postulates that, for sufficiently large Reynolds numbers, the small scale motion (SSM) is statistically independent of the large scales. It follows that the SSM should be statistically universal, locally isotropic, and described by parameters such as $v$, the kinematic viscosity, and $\langle\varepsilon\rangle$, the mean energy dissipation rate. ${ }^{1,2}$ When a scalar is introduced in a turbulent flow so that it can be treated as "passive" (i.e., it does not affect the dynamics of the flow), the rate at which it is mixed, so that a uniform concentration is eventually reached, is primarily governed by $\langle\chi\rangle$, the mean scalar dissipation rate; one therefore expects that passive scalars (e.g., temperature, humidity, and dye) should observe the same K41 postulates. ${ }^{3,4}$ If the notion that the SSM is universal were strictly valid then there would be a realistic hope for a successful statistical theory. However, growing evidence from experiments ${ }^{5-8}$ and simulations ${ }^{9,10}$ suggests that the SSM is affected by the large-scale anisotropy, which propagates down to the small scales. The SSM includes both the dissipative and inertial scales; the inertial scales will be more affected by finite Reynolds number effects than the dissipative scales. The attention paid to the SSM seems justified for the reasons outlined previously, but also in the context of developing a theory/model to predict the dispersion/mixing of a passive scalar. ${ }^{11-16}$ In this regard, the present study is carried out to further understand the mixing of velocity and scalar fluctuations in turbulence that is more closely isotropic than in earlier studies.

For isotropic turbulence at large Taylor microscale Reynolds numbers, the spectrum of $u$ is expected to exhibit a " $5 / 3$ " power-law behaviour in the inertial subrange, ${ }^{1,3}$ viz.,

$$
\phi_{u}\left(k_{1}\right)=C_{u}\langle\varepsilon\rangle^{2 / 3} k_{1}^{-5 / 3},
$$

where $\phi_{u}\left(k_{1}\right)$ is the one-dimensional spectrum $\int_{0}^{\infty} \phi_{u}\left(k_{1}\right) d k_{1}=u^{\prime 2}, k_{1}$ is the one-dimensional wavenumber; the Taylor microscale Reynolds number is defined as $R_{\lambda}=\lambda u^{\prime} / \nu$, where $\lambda$ is the 
Taylor microscale and $u$ is the streamwise-velocity fluctuation (a prime denotes the root-meansquare value). According to Sreenivasan's ${ }^{17}$ survey, the Kolmogorov constant $C_{u}$ in Eq. (1) may be considered quasi-universal, at least for $R_{\lambda} \gtrsim 50$, with a magnitude of $\sim 0.5$. For a passive scalar introduced in a turbulent flow, the scalar spectrum also exhibits a 5/3 scaling range in the inertialconvective subrange, ${ }^{3,4}$

$$
\phi_{\theta}\left(k_{1}\right)=C_{\theta}\langle\varepsilon\rangle^{-1 / 3}\langle\chi\rangle k_{1}^{-5 / 3},
$$

where $\int_{0}^{\infty} \phi_{\theta}\left(k_{1}\right) d k_{1}=\theta^{\prime 2}$ and $\theta$ is the scalar fluctuation. Sreenivasan ${ }^{18}$ has indicated that, for shear flows at $R_{\lambda}<10^{3}$, the scalar spectrum is less steep than that given by Eq. (2) and so, the ObukhovCorrsin constant $C_{\theta}$ is only defined for $R_{\lambda} \gtrsim 10^{3}$. He also indicated that for grid turbulence, where the large scales are expected not to depart significantly from isotropy, the anticipated $5 / 3$ scaling range is observed for $R_{\lambda}$ smaller than $10^{2}$. On the basis that the available sheared-turbulence and grid-turbulence data for $C_{\theta}$ seem to be scattered between 0.3 and 0.5 , Sreenivasan ${ }^{18}$ recommended an average, $R_{\lambda}$ independent, value of $\sim 0.4$.

Since the present study focuses on grid turbulence, we shall briefly discuss the findings of Sreenivasan ${ }^{17,18}$ in this context. Grid turbulence is nominally the least complex form of turbulence in view of the absence of mean shear and hence, the absence of forcing by the large scales on scales that fall in the "inertial" (or more rigorously the "scaling") and dissipative ranges. We first note that the recommendations by Sreenivasan ${ }^{17,18}$ for quasi-universal magnitudes of $C_{u}$ and $C_{\theta}$, while supposedly of some empirical value, may not accurately reflect the available information on the relative behaviour of the velocity and scalar fluctuations in grid turbulence and sheared turbulence. For example, the distinction made by Sreenivasan ${ }^{18}$ regarding the difference in behaviour of $\phi_{\theta}\left(k_{1}\right)$ between grid turbulence and sheared turbulence could equally have been made for $\phi_{u}\left(k_{1}\right)$. In fact, Antonia and Burattini ${ }^{19}$ showed that, for grid turbulence or more generally decaying-type turbulence such as on the centreline of jets and wakes, the asymptotic "4/5" law of Kolmogorov ${ }^{2}$

$$
-\left\langle(\delta u)^{3}\right\rangle=(4 / 5)\langle\varepsilon\rangle r
$$

$(\delta u=u(x+r)-u(x)$ is the velocity increment between two points separated by a distance $r)$ is approached very slowly as $R_{\lambda}$ is increased. Values of $R_{\lambda}$ in excess of $10^{4}$ may be required before an inertial subrange is actually established, i.e., the effect of "finite Reynolds number" (FRN) persists up to at least $R_{\lambda} \approx 10^{4}$. A much smaller value of $R_{\lambda}$, about $10^{3}$, appears sufficient in forced turbulence. ${ }^{19}$ The obvious implication is that Eq. (1) should be satisfied more rapidly in sheared turbulence than in grid turbulence. However, even in sheared turbulence, it is unlikely that $C_{u}$ can be estimated unambiguously when $R_{\lambda} \lesssim 10^{3}$.

The observations of Sreenivasan ${ }^{18}$ indicate that, for the case of $\phi_{\theta}(k)$, the trends are reversed, i.e., Eq. (2) is attained more quickly for grid turbulence than for sheared turbulence. However, the experimental grid-turbulence data and the numerical box-turbulence data for $-\left\langle\delta u(\delta \theta)^{2}\right\rangle$ examined by Danaila et al. ${ }^{20}$ suggest that $R_{\lambda}$ would need to exceed $10^{3}$ for the maximum value of $-\left\langle\delta u(\delta \theta)^{2}\right\rangle /(\langle\chi\rangle r)$ to reach the asymptotic "4/3" law of Yaglom ${ }^{21}$

$$
-\left\langle\delta u(\delta \theta)^{2}\right\rangle=(4 / 3)\langle\chi\rangle r
$$

$(\delta \theta=\theta(x+r)-\theta(x)$ is the temperature increment between two points separated by a distance $r)$. It is therefore unlikely, contrary to the claim by Sreenivasan ${ }^{18}$ that Eq. (2) holds for grid turbulence at $R_{\lambda}$ $<10^{3}$. This is supported by the spectral measurements of Mydlarski and Warhaft ${ }^{5,6}$ for passive-grid and active-grid flows; they have shown that, for both $\phi_{u}\left(k_{1}\right)$ and $\phi_{\theta}\left(k_{1}\right)$, there is a scaling range, where

$$
\begin{aligned}
& \phi_{u}\left(k_{1}\right) \sim k_{1}^{-m_{u}}, \\
& \phi_{\theta}\left(k_{1}\right) \sim k_{1}^{-m_{\theta}},
\end{aligned}
$$

with the magnitudes of the scaling-range exponents $m_{u}$ and $m_{\theta}$ increasing monotonically towards the asymptotic value of $5 / 3$ as $R_{\lambda}$ approaches $10^{4}$. By using Sreenivasan's ${ }^{22}$ modified form 
of Eq. (1), i.e.,

$$
\phi_{u}\left(k_{1}\right)=C_{u}{ }^{+}\langle\varepsilon\rangle^{2 / 3} k_{1}{ }^{-5 / 3}\left(k_{1} \eta\right)^{5 / 3-m_{u}}
$$

( $\eta$ is the Kolmogorov length scale), Mydlarski and Warhaft ${ }^{5}$ found that, after extrapolation, the exponent can be approximated by $m_{u}=5 / 3-a_{u} R_{\lambda}^{-2 / 3}$ with $a_{u}=5.25$. The second term on the right side (i.e., $a_{u} R_{\lambda}^{-2 / 3}$ ) represents the effect of FRN; this term is negligible when $R_{\lambda}$ approaches $10^{4}$, which is consistent with the previously mentioned FRN effect on $-\left\langle(\delta u)^{3}\right\rangle /(\langle\varepsilon\rangle r)$. From their analysis, Mydlarski and Warhaft ${ }^{5}$ showed that the scaling-range coefficient for the velocity spectrum, $C_{u}{ }^{+}\left(=4.5-2.4 m_{u}\right)$, approaches the Kolmogorov constant of $0.5\left(=C_{u}\right.$ in Eq. (1)) as $m_{u}$ approaches $5 / 3$ for increasing $R_{\lambda}$. For the scalar, $\phi_{\theta}\left(k_{1}\right)$ may be written in the form analogous to Eq. (7), viz.,

$$
\phi_{\theta}\left(k_{1}\right)=C_{\theta}{ }^{+}\langle\varepsilon\rangle^{-1 / 3}\langle\chi\rangle k_{1}^{-5 / 3}\left(k_{1} \eta\right)^{5 / 3-m_{\theta}}
$$

where the scaling-range exponent $m_{\theta}$ is closer to $5 / 3$ than $m_{u}$, and that the scaling-range coefficient for the scalar spectrum, $C_{\theta}{ }^{+}$, approaches the Obukhov-Corrsin constant, $C_{\theta}$ (in Eq. (2)), as $m_{\theta}$ approaches $5 / 3$ for increasing $R_{\lambda}$. Although a trend for $C_{\theta}{ }^{+}$as a function of $m_{\theta}$ has yet to be established, a least-squares curve fit to Mydlarski and Warhaft's ${ }^{6}$ data (their Figure 7) shows $m_{\theta}=5 / 3-a_{\theta} R_{\lambda}^{-2 / 3}$ with $a_{\theta} \approx a_{u} / 2=5.25 / 2$. This is consistent with Figure 9 of Mydlarski and Warhaft, ${ }^{6}$ where the difference between the scaling-range exponents, $m_{\theta}-m_{u} \approx\left(a_{u} / 2\right) R_{\lambda}^{-2 / 3}$, is a decreasing function of $R_{\lambda}$. The magnitude of $a_{\theta}\left(\approx a_{u} / 2\right)$ implies that the departure from 5/3 is about half as small for $\phi_{\theta}\left(k_{1}\right)$ than for $\phi_{u}\left(k_{1}\right)$. Danaila and Antonia ${ }^{23}$ (see also Danaila et al. ${ }^{20}$ ) provided an explanation for the difference between $m_{u}$ and $m_{\theta}$, or equivalently between $a_{u}$ and $a_{\theta}$, by adopting the nonlinear characteristic time $\tau(k)$ of eddies of size $k^{-1}$ ( $k$ is the magnitude of the wavenumber vector $\vec{k}$ ), first proposed by Batchelor ${ }^{24}$ and later used by Kraichnan, ${ }^{25}$ to study energy transfer in the inertial range of isotropic turbulence, viz.,

$$
\tau(k) \sim\left[\int_{0}^{k} p^{2} E(p) d p\right]^{-1 / 2},
$$

where $E(p)$ is the three-dimensional energy spectrum of the wavenumber " $p$." This characteristic time $\tau(k)$ essentially represents the effect of the cumulative strain imposed on a structure of size $k^{-1}$ by all larger structures. Danaila and Antonia ${ }^{23}$ have used this characteristic time to formulate a closure hypothesis for the nonlinear energy-transfer term in the transport equation of $E_{\theta}(k)$. This, together with the inclusion of a term reflecting the FRN effect on $E(k)$ and $E_{\theta}(k)$, has allowed them to obtain an expression for $E_{\theta}(k)$ and to compare it with $E(k)$. The analytical expression is

$$
E_{\theta}(k)=\frac{\langle\chi\rangle \tau(k)}{k} \sim \frac{1}{k}\left[\int_{0}^{k} p^{2} E(p) d p\right]^{-1 / 2} .
$$

Danaila and Antonia ${ }^{23}$ showed that, if $E(k)$ and $E_{\theta}(k)$ assume power laws similar to those described by Eqs. (5) and (6) (note that for isotropic turbulence, the three-dimensional spectra $E$ and $E_{\theta}$ should have the same slopes in the scaling range as the one-dimensional spectra $\phi_{u}$ and $\phi_{\theta}$ ), then

$$
m_{\theta}=\left(5 / 3+m_{u}\right) / 2
$$

after substituting the scaling-range expressions for $E(k)$ and $E_{\theta}(k)$ in Eq. (10), which take into account the FRN effect. Equation (11) implies that, although $m_{\theta}$ is closer to $5 / 3$ than $m_{u}$, it will reach its asymptotic value of $5 / 3$ when $m_{u}$ reaches $5 / 3$.

To provide support for Eq. (11), Danaila and Antonia ${ }^{23}$ have used data obtained from both passive and active grids. ${ }^{5,6}$ For the majority of these data, the small scales may be considered to be only approximately isotropic, as shown, for example, by the three-component vorticity measurements of Antonia et al. ${ }^{26}$ with the exception of Mydlarski and Warhaft ${ }^{6}$ - the anisotropy of the smallscale temperature fluctuations is enhanced by the presence of a mean-temperature gradient. The large scales have non-negligible departures from isotropy. The ratio of streamwise to cross-stream velocity fluctuations $u^{\prime 2} / v^{\prime 2}$ or $u^{\prime 2} / w^{\prime 2}$ in the central core region of the flow falls between 1.1 and 1.8 , and may vary slightly due to different grids. ${ }^{5,27-29}$ 
To improve isotropy at the large scales, an effective method is to introduce a short secondary contraction downstream of the grid. ${ }^{27-32}$ The overall effect is to alter the ratio of streamwise to cross-stream velocity (or vorticity) fluctuations so that it approximates 1 more closely. Comte-Bellot and Corrsin ${ }^{27}$ Lavoie ${ }^{28}$ and Lavoie et al. ${ }^{29}$ have showed that, by using a contraction with an area ratio of 1.27 and 1.36 , this reduced the anisotropy ratio $u^{\prime 2} / v^{\prime 2}$ or $u^{\prime 2} / w^{\prime 2}$ to a value somewhere between 0.9 and 1.2. Notably, with the contraction, and for a round-bar grid of $44 \%$ solidity (known as "Rd44w") with a wire helically wrapped around each bar to reduce vortex shedding, Lavoie ${ }^{28}$ found that $u^{\prime 2} / w^{\prime 2} \approx 0.99$. In Sec. II, we discuss in more detail the anisotropy ratio $\left(u^{\prime 2} / w^{\prime 2}\right)$ for the grids (including Rd44w) selected for the present study.

When a grid flow is immediately heated with a mesh of fine wires (known as a "mandoline," see Sec. II for further description) and is then slightly stretched by a short 1.36:1 contraction, we observed that the skewnesses for the scalar fluctuation and its derivative $\left(S_{\theta} \approx 0.05\right.$ and $-S_{\partial \theta / \partial x}$ $\approx 0.09$, respectively) are quite small. ${ }^{33}$ For a similar grid, with no contraction and/or with no wire wrapped on each bar, the magnitudes of $S_{\theta}$ and $-S_{\partial \theta / \partial x}$ are nearly twice as large. For the kurtosis of the scalar fluctuation and its derivative, the magnitudes of $K_{\theta}$ and $K_{\partial \theta / \partial x}$ are larger when the (1.36:1) contraction is not used. ${ }^{33}$ Expectantly, the skewness is zero if the grid turbulence is isotropic and the kurtosis (subtracted by 3 ) is zero if the distribution of the passive scalar in isotropic grid turbulence is Gaussian. The implication is that the secondary contraction improves the isotropy of the scalar fluctuations since, with the contraction, the magnitudes for both the skewness and the kurtosis are closer to zero.

For the present study, the aim is to examine the relative behaviour of the spectra $\phi_{u}\left(k_{1}\right)$ and $\phi_{\theta}\left(k_{1}\right)$ in grid turbulence with a smooth (1.36:1) contraction for which the large scales (and the small scales) are more nearly isotropic than for grid turbulence with no contraction. Of particular interest are the relative magnitudes of the spectral scaling-range exponents $m_{u}$ and $m_{\theta}$, and how closely Eq. (11) is satisfied. Also of interest are the consequent relative magnitudes of the scaling-range coefficients $C_{u}{ }^{+}$and $C_{\theta}{ }^{+}$, and the manner in which they approach $C_{u}$ (the Kolmogorov constant) and $C_{\theta}$ (the Obukhov-Corrsin constant), respectively. Although the maximum value of $R_{\lambda}$ covered by the present experiments is small (no more than $10^{2}$ ), the measurements are supplemented with available large- $R_{\lambda}\left(\approx 10^{2} \rightarrow 10^{3}\right)$ experimental data from literature and should provide a useful indication of how the asymptotic states for the velocity and temperature fields, as represented by Eqs. (1) and (2), are approached for isotropic turbulence.

\section{APPARATUS}

Figure 1 shows a schematic diagram of the wind-tunnel test section used for the present investigation. The test section includes a short (1.36:1) secondary contraction that is located at $11 M$ downstream of the grid. The design of the contraction, based on a linear theory of turbulence ${ }^{30,34}$ and the empirical data of Comte-Bellot and Corrsin, ${ }^{27}$ was carefully undertaken by Lavoie ${ }^{28}$ and Lavoie et al..$^{29}$ to improve the isotropy of the large scales and to reduce the effect of grid geometry.
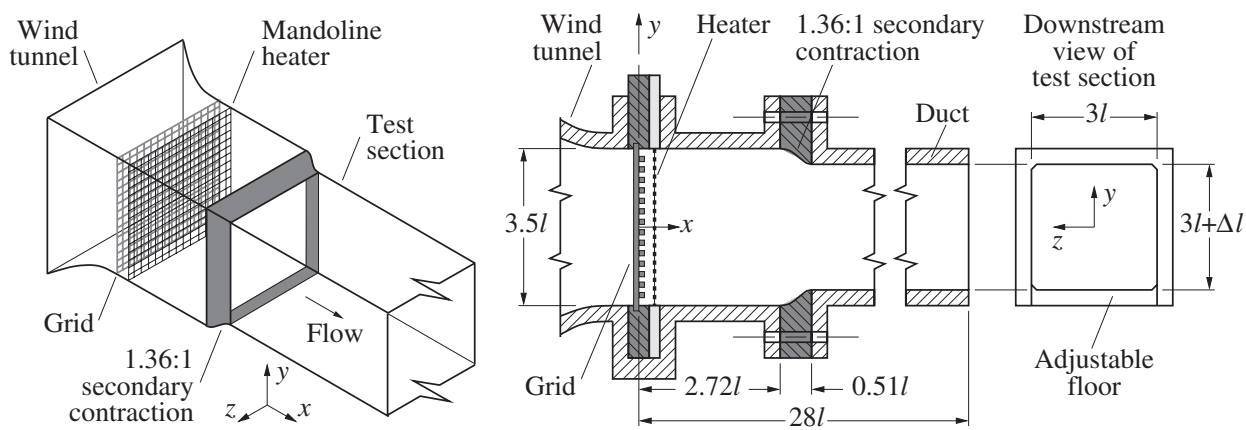

FIG. 1 . The test section of the wind tunnel. The 1.36:1 contraction is located $\approx 11 M$ downstream of the grid (scale: $l=0.1 \mathrm{~m} \approx 4 M)$. 


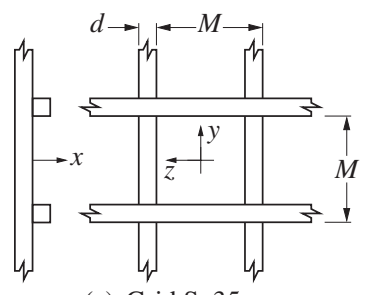

(a) Grid Sq35 $d / M=0.19 ; \sigma=0.35$

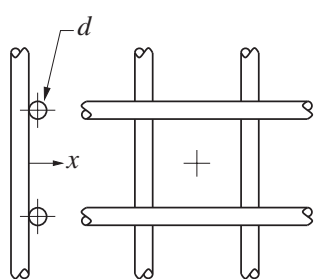

(b) Grid Rd35

$d / M=0.19 ; \sigma=0.35$

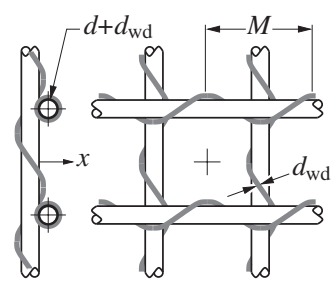

(c) Grid Rd44w

$d / M=0.26 ; \sigma=0.44 ; d_{\mathrm{wd}} \approx 0.03 M$

FIG. 2. The biplanar grid; $M=24.76 \mathrm{~mm}$. Mesh solidity is $\sigma=d / M(2-d / M) ; x$-coordinate axis lies on the centreline of the duct.

Three different biplanar grids (Figure 2) previously used by Lavoie ${ }^{28}$ and co-workers ${ }^{29,31,33}$ are used here. The first grid (Sq35) consists of square bars with a solidity ratio $(\sigma)$ of 0.35 , the second $(\mathrm{Rd} 35)$ has round bars $(\sigma=0.35)$, and the third $(\mathrm{Rd} 44 \mathrm{w})$ is made of round bars with a thin wire wrapped around each bar $(\sigma=0.44)$. For each grid, the mesh size is $M=24.76 \mathrm{~mm}$. The flow entering the grid is taken from an open-circuit (9:1 contraction) wind tunnel, and is driven by a centrifugal blower that is controlled by a variable-cycle (from 0 to $1500 \mathrm{rpm}$ ) power supply. Further details of the wind tunnel are provided in Refs. 28,29, and 33. For the majority of new data presented here, the grid-mesh Reynolds number $\left(R_{M}=M U_{\mathrm{o}} / v\right)$ is $\approx 10^{4}$, where $U_{\mathrm{o}}(\approx 6 \mathrm{~m} / \mathrm{s})$ is the mean free-stream velocity entering the grid.

In Figure 3(a), the velocity on the centreline of the wind tunnel is measured using a Pitot-static tube and a (100 Pa) micro-manometer. The velocity through the contraction part of the tunnel is not constant, and the time required for turbulence to be convected from the grid (at say location $s=0$ ) to a downstream position (at $s=x$ ) is defined by ${ }^{27-29}$

$$
t=\int_{0}^{x}\langle U(s)\rangle^{-1} d s .
$$

Equation (12) simplifies to $t=x / U_{\mathrm{o}}$ if no contraction is used. The non-dimensional time scale, $t U_{\mathrm{o}} / M$, allows direct comparison between results obtained downstream of the contraction and those obtained with no contraction. ${ }^{27-29}$ With the exception of a slight stretching of the initial grid flow in the contraction, for the most part of the duct $(\approx 80 M$ in length), there is no mean shear, no production of turbulent kinetic energy, and the turbulence simply decays. For the present results, the measurement range $20 \lesssim x / M \lesssim 100$ (or $22 \lesssim t U_{\mathrm{o}} / M \lesssim 110$ using Eq. (12)) is sufficiently far downstream of the regions of initially developing turbulence and accelerated flow in the contraction, and avoids possible effects of the duct exit (measurements furthest from the grid are $\approx 12 \mathrm{M}$ short of the exit plane of the duct). In the range $20 \lesssim x / M \lesssim 100$ shown in Figure 3(a), the time-averaged
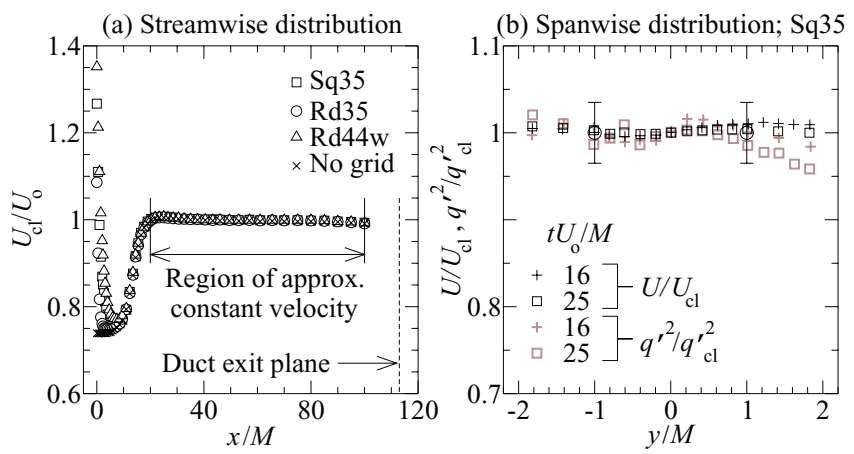

(c) Anisotropy ratio

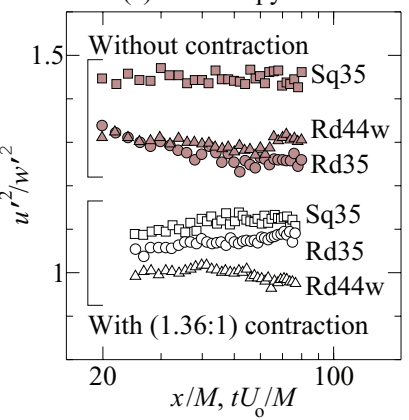

FIG. 3. (a) Centreline distribution of the wind-tunnel velocity, and Lavoie' ${ }^{28}$ measurements of (b) the velocity profile just downstream of the (1.36:1) secondary contraction and (c) the flow anisotropy on the centreline of the test section (see also Lavoie et al. ${ }^{29}$ ). 
velocity $\left(U_{\mathrm{cl}} / U_{\mathrm{o}} \approx 1 \pm 0.01\right)$ is independent of streamwise position, and the variation in wall pressure is no more than $1 \%$ of the dynamic pressure in the duct. For the same wind tunnel, Lavoie's ${ }^{28}$ crosswire measurements in the range $16 \lesssim t U_{\mathrm{o}} / M \lesssim 25$ have established that the grid flow is approximately homogeneous with spanwise velocity distribution $U(y) / U_{\mathrm{cl}} \approx 1 \pm 0.02$ and turbulent kinetic energy $q(y)^{\prime 2} / q_{\mathrm{cl}}{ }^{2} \approx 1 \pm 0.05$ (see examples in Figure 3(b); the subscript "cl" denotes the centreline value).

For clarity, we show in Figure 3(c) Lavoie's ${ }^{28}$ measurements of the anisotropy ratio $u^{\prime 2} / w^{\prime 2}$ in the duct. The improvement in isotropy with the contraction is obvious when compared with the same grid flows with no contraction. Although the effect of grid geometry persists, the difference in the anisotropy ratio, especially between the square-bar grid and the round-bar grids, is smaller. With no contraction, the anisotropy ratio is more sensitive to the geometry of the grid and, for the round bars $(\operatorname{Rd} 35$ and $\operatorname{Rd} 44 \mathrm{w}), u^{\prime 2} / w^{\prime 2}$ is dependent on $x$. Figure 3(c) shows that, with the 1.36:1 contraction, the dependence of $u^{\prime 2} / w^{\prime 2}$ on $x$ for the three grids is rather weak. The anisotropy increases slightly with $x$ for both Sq35 and Rd35; this possibly reflects the persistence of large scales produced by these grids. For Rd44w with each bar helically wrapped by a wire to disrupt the large scales, turbulence appears to relax very close to isotropy. Overall, by using the contraction to reduce the flow anisotropy, Lavoie et al. ${ }^{29}$ observed that the decay rate of turbulent kinetic energy is less dependent on grid geometry; with no contraction, the large-scale anisotropy tends to increase the magnitude of the decay rate. The decay rate and the choice of the optimum virtual origin $\left(t_{0}\right)$ are further discussed in Sec. IV.

To obtain a passive scalar, the grid flow is heated with a mesh array of 0.5 -mm-diameter Chromel-A wires located just (1.5M) downstream of the grid (see Figure 1). This method of heating is the same as that used by Zhou et al. ${ }^{35,36}$ and Antonia et al. ${ }^{37}$ (see also Danaila and Antonia ${ }^{23}$ ) to introduce the scalar in the Sq35 grid flow; it is based on the mandoline technique developed by Warhaft and Lumley. ${ }^{38}$ The horizontal and vertical wires of the mandoline heater are separated by a gap of $0.6 \mathrm{M}$ and have the same mesh size as the turbulence-generating grid. The grid flow is slightly warmer than the ambient air; a fixed temperature difference, $\Delta T \approx 2{ }^{\circ} \mathrm{C}$, is maintained by adjusting a variable-voltage (from 0 to $275 \mathrm{~V}$ ) power supply. The maximum voltage limits the grid-mesh Reynolds number $\left(R_{M}\right)$ to no more than $3 \times 10^{4}$; the maximum flow speed $\left(U_{\mathrm{o}}\right)$ obtained with the present grids is about $16 \mathrm{~m} / \mathrm{s}$. For the present measurement range of flow speeds $(\approx 6-16$ $\mathrm{m} / \mathrm{s}$ ), the pressure change across the (1.36:1) contraction is too small to produce any noticeable compressibility effects ${ }^{39}$ on the grid turbulence. The Prandtl number $(P r)$ is fixed at $\approx 0.71$. The Taylor microscale Reynolds number is in the range $10<R_{\lambda}<10^{2}$.

\section{MEASUREMENT TECHNIQUE}

For the present results, the streamwise velocity $(u)$ and the temperature $(\theta)$ fluctuations are measured simultaneously using hot and cold wires. Both wires are etched from a fine Wollaston (Pt-10\%Rh). The hot wire (diameter $d_{\text {hot }} \approx 2.50 \mu \mathrm{m}$; length $l_{\text {hot }} \approx 200 d_{\text {hot }}$ ) is operated at constant temperature with an overheat ratio of 1.5 . To minimise contamination by velocity, the cold wire $\left(d_{\text {cold }} \approx 0.63 \mu \mathrm{m} ; l_{\text {cold }} \approx 1000 d_{\text {cold }}\right)$ is operated at a constant current of $0.1 \mathrm{~mA}$ with a sensitivity of $1 \Omega /{ }^{\circ} \mathrm{C}$. To avoid interference of the measurement signals, the wires are parallel with a spanwise separation of $1 \mathrm{~mm}$ and the cold wire is located just slightly upstream of the hot wire. For the present range of measurements, the distance between the wires is 1.5-3.0 $\eta$; the wire lengths are in the range $0.6 \lesssim \eta / l_{\text {hot }} \lesssim 1.5$ and $0.5 \lesssim \eta / l_{\text {cold }} \lesssim 1.1$. Overall, the wire length-to-diameter $(l / d)$ ratios are chosen to minimise attenuation at high frequencies while maintaining $\eta / l$ as close to 1 as practicable. For $\eta / l \gtrsim 0.5$, the effect of attenuation ${ }^{40}$ underestimates $u^{\prime 2}$ by no more than $\pm 4 \%$. For a cold wire of $l / d \approx 1000$, the error ${ }^{41}$ in measuring $\theta^{\prime 2}$ is $\pm 5 \%$. The uncertainty in $\langle\varepsilon\rangle$ and $\langle\chi\rangle$, estimated by the method of propagation of errors, ${ }^{42}$ is no more than $\pm 10 \%$.

The hot-wire and cold-wire signals are digitised with a 12 bit $(200 \mathrm{kHz}) \mathrm{A} / \mathrm{D}$ converter and are low-pass filtered at half the sampling rate. To ensure that the records are of sufficient duration, we have checked that the signal fluctuations and their derivatives have indeed converged with the sample size $\left(1 \times 10^{6}\right.$ for each experimental run); the sampling period $\left(t_{\mathrm{s}} U_{\mathrm{o}} / \eta\right)$ is in the order of $10^{6}$. The power spectrum of the signal is estimated by using the Welch ${ }^{43}$ FFT method. The cutoff frequency is determined by the response time of the cold wire provided by Antonia et al. ${ }^{44}$ It is close 

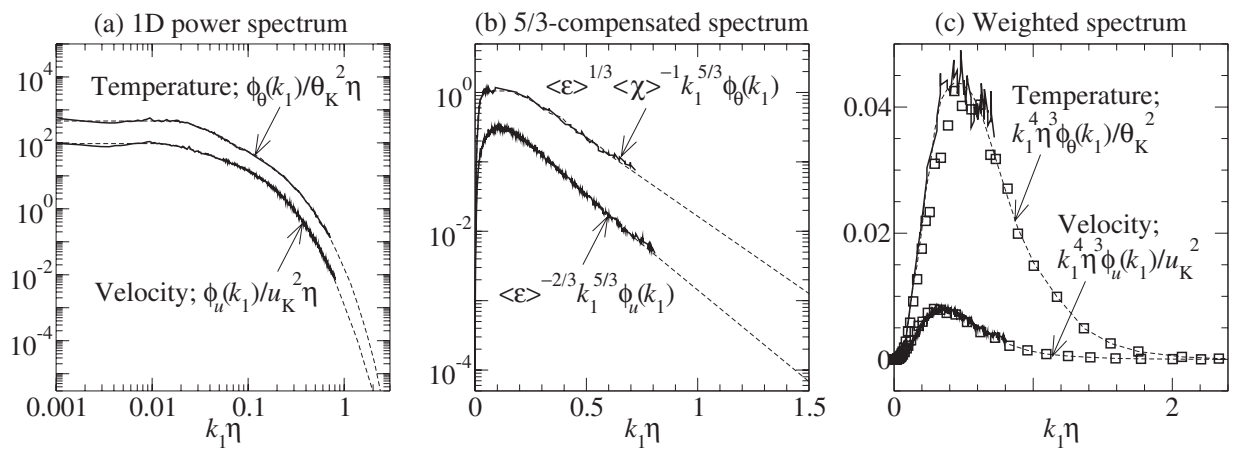

FIG. 4. Examples of curve fit and extrapolation (dashed lines) for the Kolmogorov non-dimensionalised spectrum of velocity and temperature (solid lines) (Rd44w with the contraction; $t U_{\mathrm{o}} / M \approx 70 ; R_{M} \approx 10^{4} ; R_{\lambda} \approx 40$ ). The DNS data ( $\square$ ) of Abe et al. ${ }^{47}$ and Antonia and $\mathrm{Abe}^{48}$ are for the centreline of a fully developed turbulent channel flow $\left(h^{+}=1020 ; R_{\lambda} \approx 90\right)$.

to (but usually smaller than) the Kolmogorov frequency, i.e., $0.8 \lesssim f_{\mathrm{K}}\left(2 \pi \eta / U_{\mathrm{o}}\right)<1$. While the temporal resolution is adequate, the measured spectrum is usually contaminated by electronic noise and imperfect spatial resolution of the hot and cold wires. To remove the contamination, we corrected each $u$ spectrum based on the assumption that the dissipative range of the spectrum scales on the Kolmogorov velocity and length scales. Djenidi and Antonia ${ }^{45}$ indicated that this assumption holds even when the Reynolds number is small, in contrast to the asymptotic requirement of Kolmogorov ${ }^{1,2}$ provided inhomogeneity effects are small. For convenience of curve fitting the spectrum in the dissipative range, we adopted a "model spectrum" outlined in Pope, ${ }^{46}$ and the model constants are calibrated against available data from experiments and direct numerical simulations (DNS) (e.g., see Djenidi and Antonia ${ }^{45}$ ). It should be noted that, for the purpose of extrapolation, the method of curve fit is of secondary importance provided that the experimental or DNS spectrum is sufficiently well resolved. For the passive scalar, we extended the same treatment to curve fit and extrapolate the dissipative range of the Kolmogorov-normalised $\theta$ spectrum. To check that the extrapolation provides adequate closure at the high wavenumbers, each spectrum is weighted by the wavenumber and is compared with previously published experimental and modelling data. In the examples shown in Figure 4, we included the (DNS) data of Abe et al. ${ }^{47}$ and Antonia and Abe ${ }^{48}$ for comparison with the present measurements. Review of other published data from experiments ${ }^{36}$ and modelling ${ }^{49}$ (not shown in Figure 4) indicated a similar trend at the high wavenumbers $\left(k_{1} \eta \gtrsim 0.8\right)$. The reasonable agreement between the present results and previously published data reflects the adequacy of the Kolmogorov scaling and helps to validate the extrapolation technique. The uncertainty in estimating $u^{\prime 2}$ and $\theta^{\prime 2}$ by using the spectral method, i.e., $\int_{0}^{\infty} \phi_{u}\left(k_{1}\right) d k_{1}=u^{\prime 2}$ and $\int_{0}^{\infty} \phi_{\theta}\left(k_{1}\right) d k_{1}=\theta^{\prime 2}$, is no more than $\pm 5 \%$. Truncation of the curve fits at $k_{1} \eta=1$ underestimates the derivative variances, i.e., $\int_{0}^{\infty} k_{1}^{2} \phi_{\psi}\left(k_{1}\right) d k_{1}=(\partial \psi / \partial x)^{\prime 2}$ with $\psi=u, \theta$, by no more than $10 \%$.

For the measurements shown in Figure 4 and in the next few pages, the spectra are nondimensionalised by using the Kolmogorov length $\eta=\left(v^{3} /\langle\varepsilon\rangle\right)^{1 / 4}$, velocity $u_{\mathrm{K}}=(\nu\langle\varepsilon\rangle)^{1 / 4}$, and temperature $\theta_{\mathrm{K}}=\langle\chi\rangle^{1 / 2}(\nu /\langle\varepsilon\rangle)^{1 / 4}=\left(\langle\chi\rangle \eta / u_{\mathrm{K}}\right)^{1 / 2}$. The methods for calculating $\langle\varepsilon\rangle$ and $\langle\chi\rangle$ are described in Sec. IV and the spectral results are discussed in Sec. V.

\section{THE MEAN DISSIPATION RATES}

For grid turbulence, Zhou et al. ${ }^{35,36}$ have shown that the mean dissipation rates $\langle\varepsilon\rangle$ and $\langle\chi\rangle$ are well approximated by the streamwise decay rate of the turbulent kinetic energy, defined here as $q^{\prime 2}=u^{\prime 2}+v^{\prime 2}+w^{\prime 2}$, and the temperature variance, $\theta^{\prime 2}$, respectively. From the same wind tunnel, Lavoie et al..$^{29}$ have measured the ratio $r_{u w}=u^{\prime 2} / w^{\prime 2}$ and we have made use of the axial symmetry of the flow $v^{\prime 2}=w^{\prime 2}$ to determine the turbulent kinetic energy, i.e., $q^{\prime 2}=u^{\prime 2}+2 w^{\prime 2}$. For the present grids with the contraction, Lavoie et al. ${ }^{29}$ have established that the turbulence is nearly isotropic and that both $q^{\prime 2} \sim\left(t-t_{\mathrm{o}}\right)^{n_{q}}$ and $u^{\prime 2} \sim\left(t-t_{\mathrm{o}}\right)^{n_{u}}$ exhibit a power-law rate of decay, where their decay rates are about the same, i.e., $n_{q} \approx n_{u}$. 
For the present study, the decay rates for $u^{\prime 2}$ and $\theta^{\prime 2}$ are determined from a total of 9 data points obtained in the range $22 \lesssim t U_{\mathrm{o}} / M \lesssim 110$. Figures 5 and 6 show that both $u^{\prime 2}$ and $\theta^{\prime 2}$ follow a power-law decay, where $u^{\prime 2} \sim\left(t-t_{\mathrm{o}}\right)^{n_{u}}$ and $\theta^{\prime 2} \sim\left(t-t_{\mathrm{o}}\right)^{n_{\theta}}$ with the power-law exponents $n_{u}, n_{\theta}$ $<0$. The mean dissipation rates are defined as

$$
\begin{aligned}
& \left\langle\varepsilon_{\mathrm{d}}\right\rangle=-\frac{1}{2} \frac{d q^{\prime 2}}{d t}=-\frac{1}{2}\left(1+\frac{2}{r_{u w}}\right) \frac{d u^{\prime 2}}{d t}, \\
& \left\langle\chi_{\mathrm{d}}\right\rangle=-\frac{1}{2} \frac{d \theta^{\prime 2}}{d t},
\end{aligned}
$$

where each data point is calculated by using the 3-point centre-difference scheme and then averaged over its two closest points. To avoid end effects due to the scheme, the data point on each end of a batch of 9 points is removed. Taking the time derivative of the power laws for Eqs. (13) and (14) gives the relations

$$
\begin{gathered}
\left\langle\varepsilon_{\mathrm{d}}\right\rangle \sim-\frac{1}{2}\left(1+\frac{2}{r_{u w}}\right) n_{u}\left(t-t_{\mathrm{o}}\right)^{n_{u}-1}, \\
\left\langle\chi_{\mathrm{d}}\right\rangle \sim-\frac{1}{2} n_{\theta}\left(t-t_{\mathrm{o}}\right)^{n_{\theta}-1} .
\end{gathered}
$$

The virtual origin, $t_{\mathrm{o}}$, is selected so that both $n_{u}$ and $n_{\theta}$ are independent of the measurement range $22 \lesssim t U_{\mathrm{o}} / M \lesssim 110$ (using Eq. (12)) and should account for the effect of the secondary contraction. If no contraction is used, the correction for the virtual origin is $t-t_{\mathrm{o}}=\left(x-x_{\mathrm{o}}\right) / U_{\mathrm{o}}$ by assuming Taylor's hypothesis. Since the mandoline (source of heat) is introduced immediately downstream of the grid (source of turbulence), $t_{\mathrm{o}}$ should be about the same for both the velocity and passive-temperature fields. The optimum value for $t_{\mathrm{o}}$ is determined by the method described by Lavoie et al. ${ }^{29}$ and Antonia et $a l .{ }^{37}$ which involves the Taylor microscale $(\lambda)$ and the Corrsin microscale $\left(\lambda_{\theta}\right)$. The microscales are defined as

$$
\begin{gathered}
\lambda^{2} \sim v\left(q^{\prime 2} /\left\langle\varepsilon_{\mathrm{d}}\right\rangle\right) \sim\left(-v / n_{u}\right)\left(t-t_{\mathrm{o}}\right), \\
\lambda_{\theta}{ }^{2} \sim \kappa\left(\theta^{\prime 2} /\left\langle\chi_{\mathrm{d}}\right\rangle\right) \sim\left(-\kappa / n_{\theta}\right)\left(t-t_{\mathrm{o}}\right),
\end{gathered}
$$

where $\lambda^{2} /\left(t-t_{\mathrm{o}}\right) \sim\left(-v / n_{u}\right)$ and $\lambda_{\theta}{ }^{2} /\left(t-t_{\mathrm{o}}\right) \sim\left(-\kappa / n_{\theta}\right)$ should be constant in the power-law decay range. ${ }^{29,37}$ For each grid flow shown in Figures 5 and 6 , we find that the same optimum value of $t_{\mathrm{o}}$ satisfies both the velocity and temperature measurements. Varying $t_{\mathrm{o}} U_{\mathrm{o}} / M$ by \pm 1.0 changes $n_{u}$ and $n_{\theta}$ by no more than \pm 0.03 . For the curve fits in Figures 5 and 6 , the $90 \%$ confidence limit for $n_{u}$ and
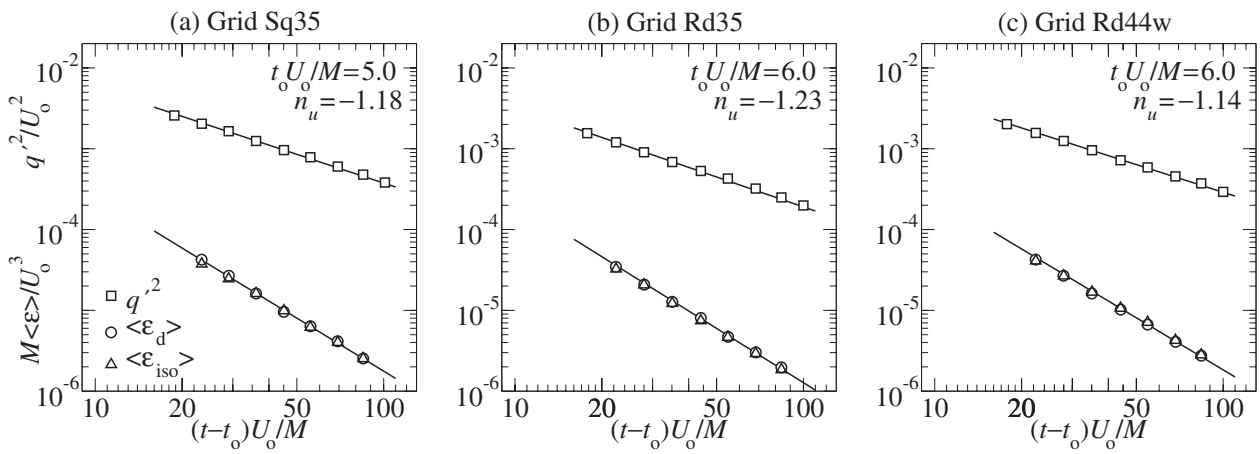

FIG. 5. The power-law decay of turbulent kinetic energy, $q^{\prime 2}=u^{\prime 2}+2 w^{\prime 2}$, and its mean dissipation rate, $\langle\varepsilon\rangle$, downstream of each grid with adjustment for optimum virtual origin $t_{\mathrm{o}} U_{\mathrm{o}} / M . u^{\prime 2} / w^{\prime 2} \approx 1.11(\mathrm{Sq} 35), \approx 1.07(\operatorname{Rd} 35)$ and $\approx 0.99(\operatorname{Rd} 44 \mathrm{w})$. $n_{u}$ is the decay exponent; $R_{M} \approx 10^{4}$. 

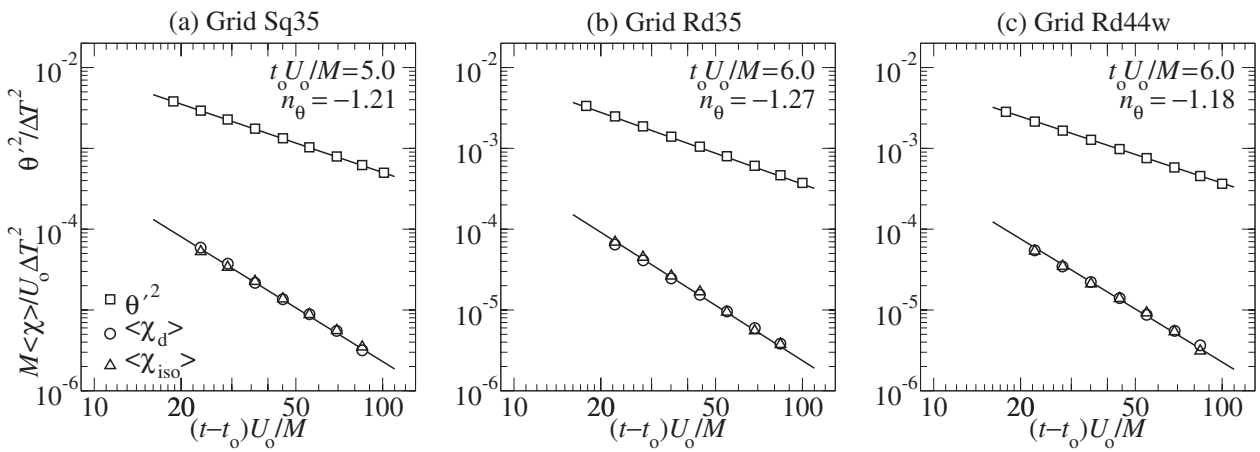

FIG. 6. The power-law decay of temperature variance, $\theta^{\prime 2}$, and its mean dissipation rate, $\langle\chi\rangle$, downstream of each grid with adjustment for optimum virtual origin $t_{\mathrm{o}} U_{\mathrm{o}} / M . n_{\theta}$ is the decay exponent; $R_{M} \approx 10^{4}$.

$n_{\theta}$ is \pm 0.03 . In Figure 7(a), the ratio between the microscales,

$$
\left(\frac{\lambda}{\lambda_{\theta}}\right)^{2} \sim \operatorname{Pr} \frac{q^{\prime 2} /\left\langle\varepsilon_{\mathrm{d}}\right\rangle}{\theta^{\prime 2} /\left\langle\chi_{\mathrm{d}}\right\rangle} \sim \operatorname{Pr} \frac{\tau}{\tau_{\theta}},
$$

is proportional to the velocity/scalar time scale ratio $\tau / \tau_{\theta}$. For each grid, $\tau / \tau_{\theta}(\approx 1 \pm 0.1)$ is reasonably independent of the range of the measurements. Since both $q^{\prime 2}$ and $\theta^{\prime 2}$ decay according to a power law, we have checked that the time scale ratio indeed closely matches the ratio between the temperature and the velocity power-law exponents, i.e., $\tau / \tau_{\theta} \approx n_{\theta} / n_{u}$. Inspection of Table I shows that, for the three grids, the ratio $n_{\theta} / n_{u}$ is from $\approx 1.03$ to 1.04 .

Since decaying grid turbulence is an approximation to isotropic turbulence, comparison between the "decay" dissipation rates and the "isotropic" dissipation rates should serve as a measure of the flow anisotropy. For homogeneous isotropic turbulence, the mean dissipation rates are defined by the wavenumber-weighted spectrum of $u$ and $\theta$, viz.,

$$
\begin{gathered}
\left\langle\varepsilon_{\text {iso }}\right\rangle=15 v \int_{0}^{\infty} k_{1}^{2} \phi_{u}\left(k_{1}\right) d k_{1}, \\
\left\langle\chi_{\text {iso }}\right\rangle=3 \kappa \int_{0}^{\infty} k_{1}^{2} \phi_{\theta}\left(k_{1}\right) d k_{1} .
\end{gathered}
$$

Figures 7(b) and 7(c) show that, for each grid, the flow is very nearly isotropic, where the decay/isotropic ratios for $\left\langle\varepsilon_{\mathrm{d}}\right\rangle /\left\langle\varepsilon_{\text {iso }}\right\rangle$ and $\left\langle\chi_{\mathrm{d}}\right\rangle /\left\langle\chi_{\text {iso }}\right\rangle$ are $\approx 1 \pm 0.1$. The decay/isotropic ratios for the Kolmogorov length $\eta_{\mathrm{d}} / \eta_{\text {iso }}$, velocity $u_{\mathrm{K}} / u_{\mathrm{K}}$ iso , and temperature $\theta_{\mathrm{Kd}} / \theta_{\mathrm{K}}$ iso are $\approx 1 \pm 0.03$. The close
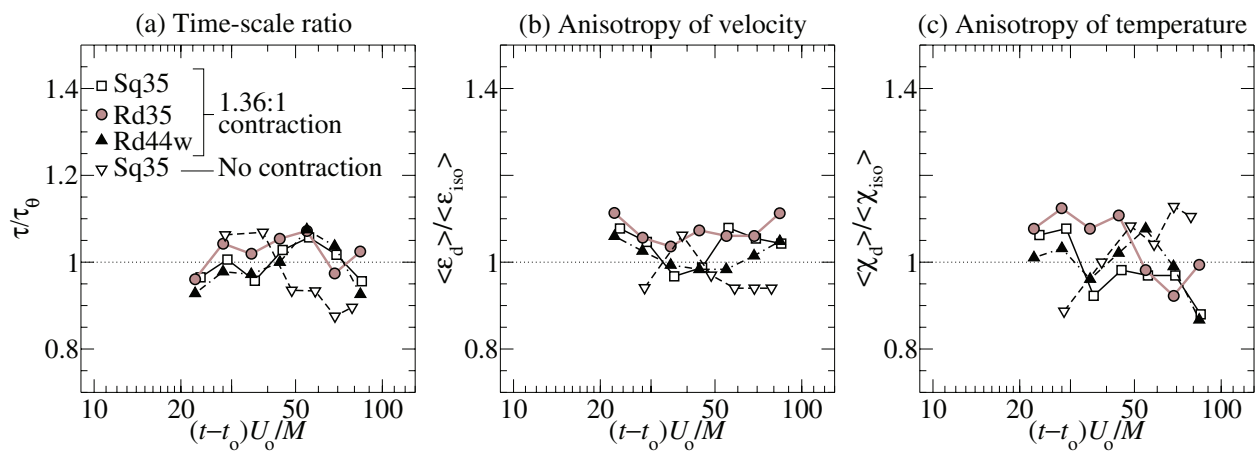

FIG. 7. (a) The time scale ratio, $\tau / \tau_{\theta}$, and the anisotropy of the estimated mean dissipation rates (b) $\left\langle\varepsilon_{\mathrm{d}}\right\rangle /\left\langle\varepsilon_{\text {iso }}\right\rangle$, and (c) $\left\langle\chi_{\mathrm{d}}\right\rangle /\left\langle\chi_{\text {iso }}\right\rangle$ from the data in Figures 5 and 6. The present data are with the contraction; the data with no contraction are from Zhou et al. $^{35}$ and Antonia et al. ${ }^{37}$ 
TABLE I. Review of decay exponents $\left(n_{u}\right.$ and $\left.n_{\theta}\right)$ of different grid flows from the same wind tunnel with and without the (secondary) contraction. The grid is at $t U_{\mathrm{o}} / M=0 ; M=24.76 \mathrm{~mm}$. The "microscale" method is used to determine the optimum virtual origin $\left(t_{\mathrm{o}} U_{\mathrm{o}} / M\right)$ for measurements in the range $22<t U_{\mathrm{o}} / M<110 ; r_{u w}=u^{\prime 2} / w^{\prime 2}$ is the anisotropy ratio. For the measurements of $n_{\theta}$, the "mandoline" heating method is used; $\Delta T \approx 2{ }^{\circ} \mathrm{C}$. For the present measurements, the $90 \%$ confidence limit for both $n_{u}$ and $n_{\theta}$ is $\pm 0.03 . R_{M} \approx 10^{4}$.

\begin{tabular}{llccccc}
\hline \hline Reference & Grid & $t_{\mathrm{o}} U_{\mathrm{o}} / M$ & $-n_{u}$ & $-n_{\theta}$ & $r_{u w}$ & Initial condition \\
\hline Antonia et al. $^{37}$ & $\mathrm{Sq} 35$ & 0 & 1.33 & 1.37 & $/$ & No contraction \\
Lavoie $^{28}$ & $\mathrm{Sq} 35$ & 7.0 & 1.05 & $/$ & 1.45 & No contraction \\
& $\mathrm{Rd} 35$ & 6.0 & 1.19 & $/$ & 1.27 & \\
& $\mathrm{Rd} 44 \mathrm{w}$ & 6.0 & 1.18 & $/$ & 1.30 & \\
Lavoie et al. & & & & & & \\
& $\mathrm{Sq} 35$ & 5.0 & 1.18 & $/$ & 1.11 & $1.36: 1$ contraction \\
& $\mathrm{Rd} 35$ & 5.0 & 1.22 & $/$ & 1.07 & \\
Present & $\mathrm{Rd} 44 \mathrm{w}$ & 7.0 & 1.10 & $/$ & 0.99 & \\
measurements & $\mathrm{Sq} 35$ & 5.0 & 1.18 & 1.21 & $/$ & \\
& $\mathrm{Rd} 35$ & 6.0 & 1.23 & 1.27 & $/$ & \\
& $\mathrm{Rd} 44 \mathrm{w}$ & 6.0 & 1.14 & 1.18 & $/$ & \\
\hline \hline
\end{tabular}

agreement between the "decay" and "isotropic" measurements lends support to the extrapolation technique discussed in Sec. III.

A review summary of results in Table I shows that, when isotropy is improved with the contraction (i.e., $r_{u w}=u^{\prime 2} / w^{\prime 2}$ is closer to 1 ), the magnitude of $n_{u}$ is less different between each grid. For an optimum virtual origin, however, the magnitude of $n_{\theta}$ is slightly larger than that of $n_{u}$; for for Rd44w that produces the most isotropic flow, $n_{\theta} / n_{u}$ is not exactly equal to 1 . This evidence may suggest that the passive-scalar field, although mixed and advected by a very nearly isotropic flow, does not behave exactly as the velocity field. In Sec. V, the differences between the two fields, as identified in their power spectra, show that their characteristics are indeed not the same.

\section{THE POWER SPECTRUM}

Figure 8(a) shows typical spectra of velocity $(u)$ and temperature $(\theta)$ for a grid flow (Sq35) stretched by the secondary (1.36:1) contraction. The other grid flows (Rd35 and Rd44w) stretched by the contraction produce very similar spectra, i.e., the grid geometry has negligible effect on the spectral scaling range. However, for each grid flow, there is a significant scaling range for $\phi_{\theta}$ but not for $\phi_{u}$. This difference is observed in Figures 8(a) and 8(b) with and without the contraction, respectively; it is a feature of grid turbulence at low Reynolds numbers $R_{\lambda} \lesssim 10^{2}$ (e.g., Sepri5 ${ }^{50}$ and Rey and Boudjemaa ${ }^{51}$ ). Mydlarski and Warhaft ${ }^{5,6}$ showed that, by increasing $R_{\lambda}$, this widens
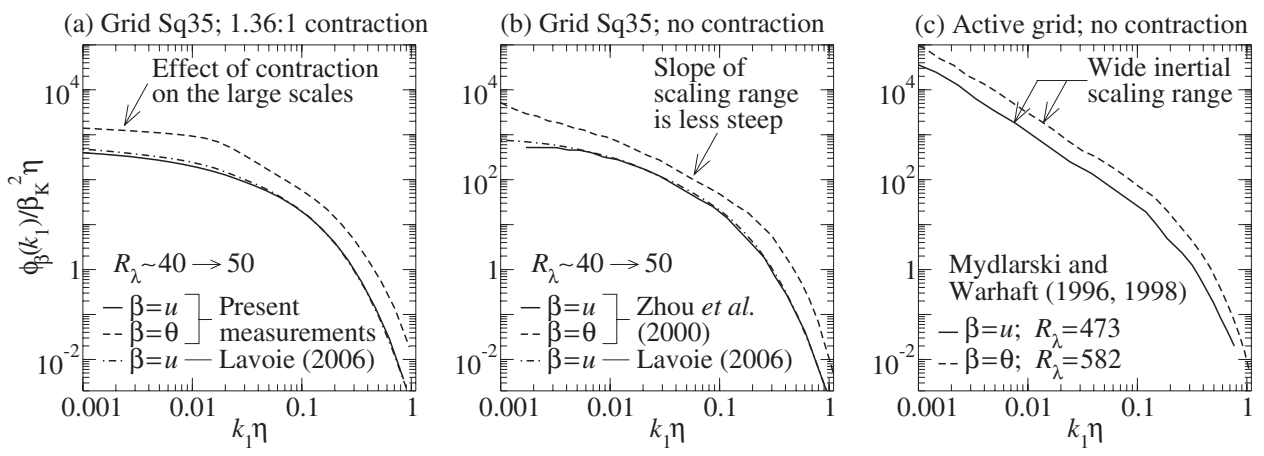

FIG. 8. Effect of inlet conditions on the Kolmogorov non-dimensionalised velocity $(u)$ and temperature $(\theta)$ spectra; $(a, b)$ grid Sq35 with and without the contraction; $t U_{\mathrm{o}} / M \approx 30 \rightarrow 40$ (present measurements, Zhou et al. ${ }^{35}$ and Lavoie ${ }^{28}$ ); (c) active grid with no contraction; $t U_{\mathrm{o}} / M \approx 70$ (Mydlarski and Warhaft ${ }^{5,6}$ ). 

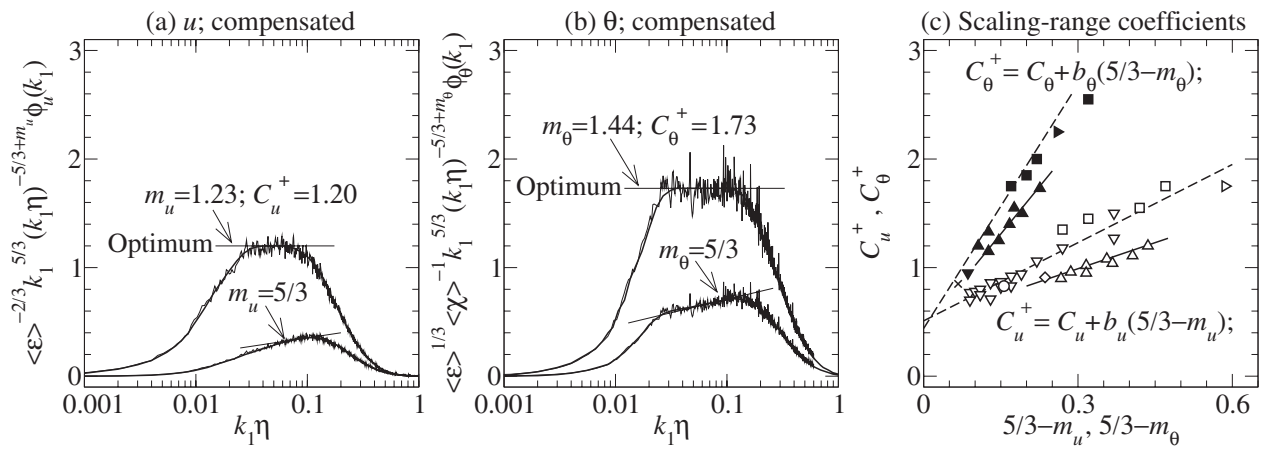

FIG. 9. (a, b) Examples of compensated spectrum (present data, Rd44w with the $1.36: 1$ contraction; $t U_{\mathrm{o}} / M \approx 70 ; R_{\lambda}$ $\approx 35$ ). (c) The coefficients for optimum compensation $\left(10<R_{\lambda}<10^{3}\right)$; the measurements $(u \Delta, \theta \mathbf{\Delta})$ are for Rd44w with the contraction. For the Sq35 data $(u \square, \theta \mathbf{\square})$ and the data of Mydlarski and Warhaft ${ }^{5}(u \nabla)$ and Yeh and Van $\operatorname{Atta}^{52}(u \triangleright, \theta$ $\triangleright$ ), the grid flows are not stretched by a contraction. The velocity data points $\diamond, \circ$, and $\times$ are obtained from Figure 10(b). The temperature data point $\mathbf{\nabla}$ is obtained from Figure 10(c).

the scaling range and, for $R_{\lambda}>10^{2}$, both $\phi_{u}$ and $\phi_{\theta}$ exhibit nearly the same wide scaling range (Figure 8(c)).

Figures 9(a) and 9(b) show examples of $u$ and $\theta$ spectra, which are "optimally" compensated:

$$
\begin{aligned}
& C_{u}{ }^{+}\left(k_{1}\right)=\langle\varepsilon\rangle^{-2 / 3} k_{1}{ }^{5 / 3}\left(k_{1} \eta\right)^{-5 / 3+m_{u}} \phi_{u}\left(k_{1}\right), \\
& C_{\theta}{ }^{+}\left(k_{1}\right)=\langle\varepsilon\rangle^{1 / 3}\langle\chi\rangle^{-1} k_{1}{ }^{5 / 3}\left(k_{1} \eta\right)^{-5 / 3+m_{\theta}} \phi_{\theta}\left(k_{1}\right),
\end{aligned}
$$

compared with those which are " $m_{u}=m_{\theta}=5 / 3$ " compensated:

$$
\begin{aligned}
& C_{u}\left(k_{1}\right)=\langle\varepsilon\rangle^{-2 / 3} k_{1}{ }^{5 / 3} \phi_{u}\left(k_{1}\right), \\
& C_{\theta}\left(k_{1}\right)=\langle\varepsilon\rangle^{1 / 3}\langle\chi\rangle^{-1} k_{1}{ }^{5 / 3} \phi_{\theta}\left(k_{1}\right) .
\end{aligned}
$$

For the optimally compensated spectra, the scaling-range exponents, i.e., $0 \ll\left(m_{u}, m_{\theta}\right)<5 / 3$ and $m_{u}<m_{\theta}$, are selected by a method of trial-and-error to produce the largest plateaux in the spectra. The results are shown in Figure 9(c), where the scaling-range coefficients $C_{u}{ }^{+}$and $C_{\theta}{ }^{+}$ are the maximum values corresponding to their respective, optimally compensated, power spectra. Changing the scaling-range exponents $m_{u}$ and $m_{\theta}$ by $\pm 5 \%$ generally alters the respective coefficients $C_{u}{ }^{+}$and $C_{\theta}{ }^{+}$by no more than $\pm 10 \%$. The uncertainty in the estimates of the plateaux $C_{u}{ }^{+}$and $C_{\theta}{ }^{+}$ is about $\pm 10 \%$.

To supplement the present data, we have included in Figure 9(c) the measurements of $\mathrm{C}_{u}{ }^{+}$ obtained from Yeh and Van Atta ${ }^{52}$ (their Figures 2 and 3; $x / M=35$ ) and those reported by Mydlarski and Warhaft ${ }^{5}$ (their Figure 11). Further examples of $C_{u}{ }^{+}$and $C_{\theta}{ }^{+}$are determined from the spectra shown in Figure 10; the optimally compensated spectra are recalculated from the 5/3 compensated spectra reported by Schedvin et al. ${ }^{53}$ (their Figure 8 includes the data of Kistler and Vrebalovich ${ }^{54}$ and Comte-Bellot and Corrsin ${ }^{55}$ ) and Mydlarski and Warhaft ${ }^{6}$ (their Figure 6).

Inspection of Figures 9(a), 9(b), and 10 clearly shows that the scaling ranges for both the velocity and the temperature spectra do not exhibit a $5 / 3$ slope (for $R_{\lambda}<10^{3}$ ). Instead, the scaling ranges (represented by the largest spectral plateaux) indicate that the slopes are less steep, i.e., $5 / 3-m_{u}$ and $5 / 3-m_{\theta}$. This is consistent with Eqs. (7) and (8), where the scaling-range coefficients $C_{u}{ }^{+}$and $C_{\theta}{ }^{+}$are not expected to match the constants $C_{u}$ and $C_{\theta}$.

Figure 9(c) shows that $C_{u}{ }^{+}$and $C_{\theta}{ }^{+}$increase approximately linearly with $5 / 3-m_{u}$ and $5 / 3-m_{\theta}$, respectively. Although both the velocity and temperature scaling ranges are sensitive to the contraction, they do not behave the same. In terms of the magnitude and the rate-of-change, the contraction tends to affect $C_{u}{ }^{+}$more than $C_{\theta}{ }^{+}$. For the same magnitude $\left(m_{u}=m_{\theta}\right)$ of the optimum scaling-range exponents, $C_{u}{ }^{+}<C_{\theta}{ }^{+}$. 

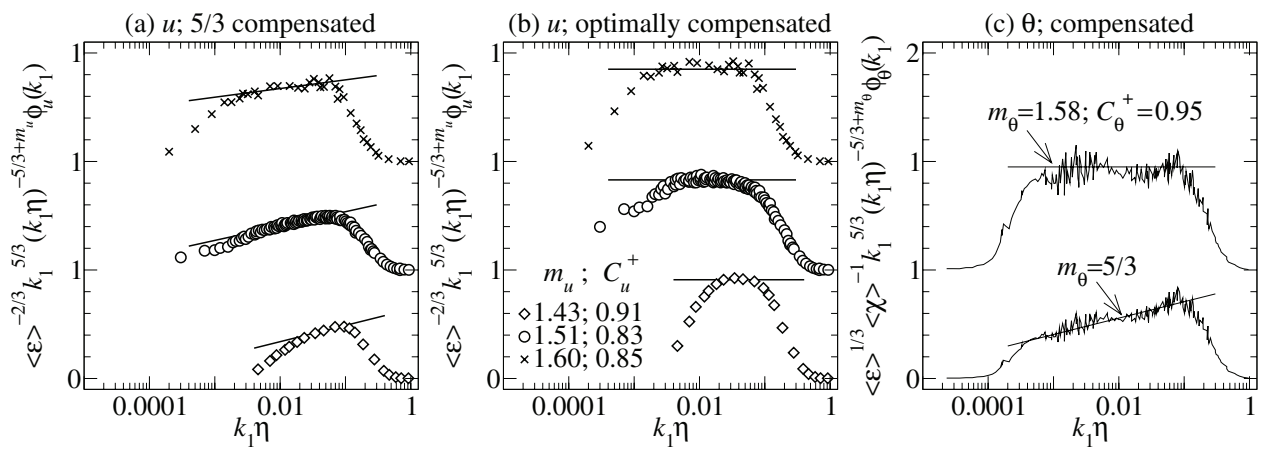

FIG. 10. Review of spectral data with the effect of compensation; (a, b) velocity; with contraction $\diamond R_{\lambda} \sim 40 \rightarrow 50$ (ComteBellot and Corrsin ${ }^{55}$ ); no contraction $\circ R_{\lambda}=280\left(\right.$ Schedvin et al.$^{53}$ ); no contraction $\times R_{\lambda}=670$ (Kistler and Vrebalovich ${ }^{54}$ ); (c) temperature; no contraction $R_{\lambda}=582$ (Mydlarski and Warhaft ${ }^{6}$ ). The spectra (from bottom to top) are vertically offset by 0,1 , and 2 , respectively.

In Figure 11, the scaling-range exponents are plotted in terms of the Reynolds number $R_{\lambda}$. For each grid (Sq35, Rd35, and Rd44w) with the 1.36:1 contraction, the error bars indicate small variations obtained for a fixed speed $\left(R_{M} \approx 10^{4}\right)$ over the region $22 \lesssim t U_{\mathrm{o}} / M \lesssim 110$. Figure 11 shows that, with this contraction, the scaling-range exponents are about the same for all three grids. This evidence lends support to Lavoie's ${ }^{28}$ deduction that the effect of grid geometry is rather small with the present contraction.

Since Rd44w produces the most isotropic turbulence (Figure 3(c)) and the decay/isotropic ratios $\left\langle\varepsilon_{\mathrm{d}}\right\rangle /\left\langle\varepsilon_{\text {iso }}\right\rangle$ and $\left\langle\chi_{\mathrm{d}}\right\rangle /\left\langle\chi_{\text {iso }}\right\rangle$ are $\approx 1 \pm 0.1$ (see Sec. IV), we decided to test different flow speeds (i.e., $\approx 6-16 \mathrm{~m} / \mathrm{s}$; see Sec. II) for a fixed downstream distance $\left(t U_{\mathrm{o}} / M \approx 70\right)$ from this grid to obtain a wider range of Reynolds number $\left(30<R_{\lambda}<60\right.$ ). These measurements (for Rd44w) are shown as triangular symbols $(\triangle, \mathbf{\Delta})$ in Figures 9(c) and 11-14; the Rd44w spectra for $u$ and $\theta$ are given in Figure 15(a).

Figures 11 and 12 show that the scaling-range exponents vary with $R_{\lambda}$; the scaling range widens as $R_{\lambda}$ is increased (Figure 15(a)). According to Mydlarski and Warhaft's ${ }^{5}$ results, the Reynoldsnumber variation reasonably follows a " $-2 / 3$ " power law (i.e., $m_{u} \propto R_{\lambda}^{-2 / 3}$ ) and that a $5 / 3$ scaling range is not observed until $R_{\lambda} \gtrsim 10^{4}$. The formulas used to describe the scaling-range exponents, after Danaila and Antonia, ${ }^{23}$ may be written as

$$
\begin{aligned}
& m_{u}=5 / 3-a_{u} R_{\lambda}^{-2 / 3}, \\
& m_{\theta}=\left(5 / 3+m_{u}\right)\left(a_{\theta} / a_{u}\right)=(5 / 3)\left(2 a_{\theta} / a_{u}\right)-a_{\theta} R_{\lambda}^{-2 / 3} .
\end{aligned}
$$

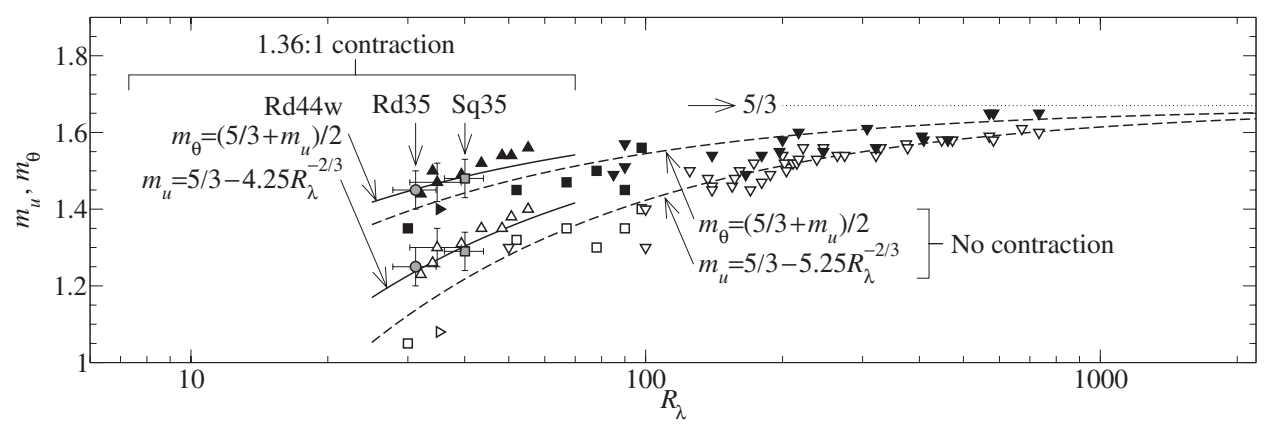

FIG. 11. The scaling-range exponents $m_{u}$ and $m_{\theta}$ as functions of $R_{\lambda}$. The present measurements are mostly for Rd44w with the contraction $(u \triangle, \theta \mathbf{\Delta})$; for each grid flow (Sq35, Rd35, and $\mathrm{Rd} 44 \mathrm{w}$ ), the error bars represent measurements over the range $t U_{\mathrm{o}} / M \approx 22 \rightarrow 110$ at a fixed $R_{M}\left(\approx 10^{4}\right)$. For the data of Danaila and $\operatorname{Antonia}^{23}(u \square, \theta \mathbf{\square})$, Mydlarski and Warhaft ${ }^{5}(u \nabla$, $\theta \boldsymbol{\nabla})$, and Yeh and Van Atta ${ }^{52}(u \triangleright, \theta \triangleright)$, the grid flow is not stretched by a contraction. See Table II for a summary of the curve fits. 


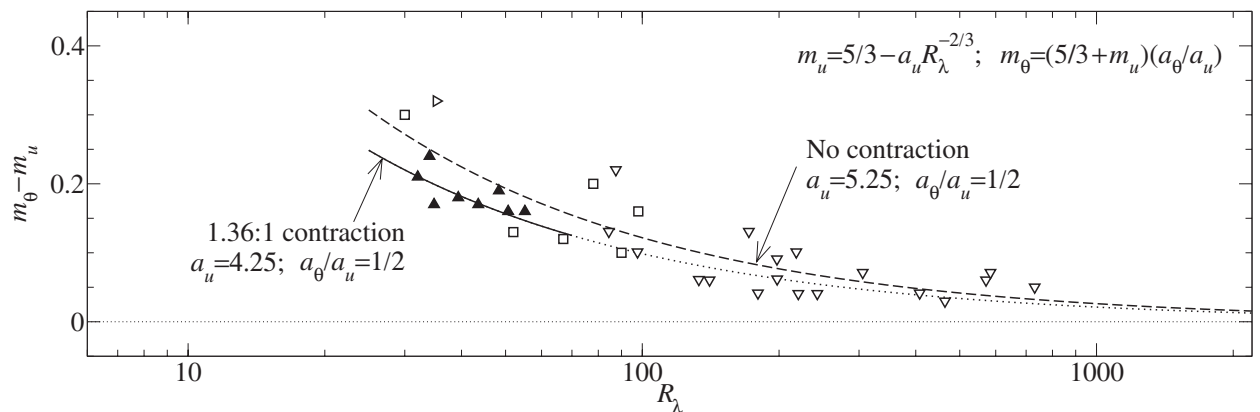

FIG. 12. The difference between the scaling-range exponents, $m_{\theta}-m_{u}$, as a function of $R_{\lambda}$. The present measurements with the contraction are shown as $\boldsymbol{\Delta}(\mathrm{Rd} 44 \mathrm{w})$. With no contraction, the data are shown as $\square$ (Danaila and Antonia ${ }^{23}$ ), $\nabla$ (Mydlarski and Warhaft $\left.{ }^{5}\right)$, and $\triangleright\left(\right.$ Yeh and Van $\left.\mathrm{Atta}^{52}\right)$.

Given that the scaling-range coefficients in Figure 9(c) have a linear dependence on the scaling-range exponents, we have adopted the following expressions, after Mydlarski and Warhaft: ${ }^{5}$

$$
\begin{gathered}
C_{u}{ }^{+}=C_{u}+b_{u}\left(5 / 3-m_{u}\right), \\
C_{\theta}{ }^{+}=C_{\theta}+b_{\theta}\left(5 / 3-m_{\theta}\right) .
\end{gathered}
$$

Using Eqs. (26) and (27), the scaling-range coefficients are expressed in terms of $R_{\lambda}$ :

$$
\begin{gathered}
C_{u}{ }^{+}=C_{u}+a_{u} b_{u} R_{\lambda}{ }^{-2 / 3}, \\
C_{\theta}{ }^{+}=C_{\theta}+5 / 3\left(1-2 a_{\theta} / a_{u}\right) b_{\theta}+a_{\theta} b_{\theta} R_{\lambda}{ }^{-2 / 3},
\end{gathered}
$$

where the empirical coefficients $\left(a_{u}, a_{\theta}, b_{u}, b_{\theta}, C_{u}\right.$, and $\left.C_{\theta}\right)$ are determined from the available experimental data.

In Figure 11, the least-squares curve fits to the data using Eqs. (26) and (27) yield $a_{u} / a_{\theta} \approx 2.0$ \pm 0.02 , where the rms difference is no more than $\pm 5 \%$. From this, it naturally follows that

$$
m_{\theta}-m_{u} \approx\left(a_{u} / 2\right) R_{\lambda}^{-2 / 3} .
$$

Figure 12 shows that, for Reynolds number in the range $R_{\lambda} \lesssim 10^{2}$, the difference between the scaling-range exponents $\left(m_{\theta}-m_{u}\right)$ is non-negligible; both the scatter in the data and the magnitude of $a_{u}(=4.25)$ with the $1.36: 1$ contraction appear slightly smaller than those with no contraction. Overall, least-squares curve fits of the form given by Eq. (32) are broadly consistent with the trends of the available data, and the rms difference is no more than $\pm 20 \%$. Note that, for the measurements obtained with no contraction, the value $a_{u}=5.25$ matches that reported by Mydlarski and Warhaft. ${ }^{5}$ According to Figure 12, as $m_{\theta}-m_{u}$ tends to zero for $R_{\lambda}>10^{2}$, any effects due to different initial conditions are likely to diminish and/or are not expected to be easily distinguishable.

Figure 13 shows the scaling-range coefficients $\left(C_{u}{ }^{+}\right.$and $\left.C_{\theta}{ }^{+}\right)$plotted in terms of $R_{\lambda}$. Since $R_{\lambda}$ is small with the available measurements (especially for $C_{\theta}{ }^{+}$), we have adopted the "universal" constants $C_{u} \sim 0.5$ and $C_{\theta} \sim 0.4$ recommended by Sreenivasan ${ }^{17,18}$ to provide the initial curve fits using Eqs. (28)-(31). The coefficients $\left(b_{u}, b_{\theta}, C_{u}\right.$, and $\left.C_{\theta}\right)$ are then reiterated, by trial-and-error, to minimise the rms difference. For each curve fit shown in Figures 9(c) and 13, the rms difference is no more than $\pm 6 \%$. Figure 13 shows that the optimised curve fit for $C_{u}{ }^{+}$with no contraction is in good agreement with the measurements of Schedvin et al. ${ }^{53}$ Kistler and Vrebalovich, ${ }^{54}$ and Saddoughi and Veeravalli, ${ }^{56}$ obtained for large Reynolds numbers in the range $10^{2}<R_{\lambda}<10^{3}$ (indicated by the error bars in Figure 13).

From Eqs. (30) and (31), and with $a_{u} / a_{\theta} \approx 2.0$ (from the curve fits in Figures 11 and 12), it follows that

$$
C_{\theta}{ }^{+}-C_{u}{ }^{+} \approx C_{\theta}-C_{u}+\left(a_{\theta} b_{\theta}-a_{u} b_{u}\right) R_{\lambda}{ }^{-2 / 3} .
$$




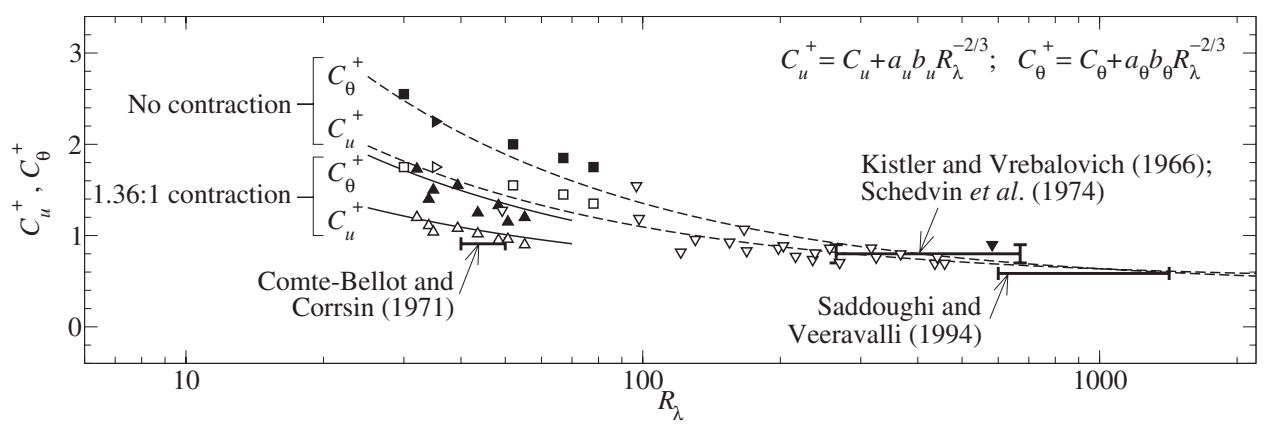

FIG. 13. The scaling-range coefficients $C_{u}{ }^{+}$and $C_{\theta}{ }^{+}$as functions of $R_{\lambda}$. The symbols are as defined in Figure 11. For the curve fits (solid and dashed lines), the empirical coefficients are summarised in Table II. The error bars indicate the approximate loci of other velocity measurements obtained with a slight $(1.27: 1)$ contraction (Comte-Bellot and Corrsin ${ }^{55}$ ) and with no contraction (Kistler and Vrebalovich, ${ }^{54}$ Schedvin et al. ${ }^{53}$ Saddoughi and Veeravalli ${ }^{56}$ ).

Although there are rather few data points in Figure 14, it is evident that the magnitude of $C_{\theta}{ }^{+}-C_{u}{ }^{+}$ is systematically smaller with the $1.36: 1$ contraction than with no contraction. The least-squares fit of the form given by Eq. (33) confirms this trend; for each curve fit, the rms difference is $\sim \pm 20 \%$. The uncertainty in measuring $C_{\theta}{ }^{+}-C_{u}{ }^{+}$from the spectra is about $\pm 20 \%$ and the apparent difference between the trends in Figure 14 cannot be attributed to scatter. Attempts to improve the curve fits would require more experimental data points at higher $R_{\lambda}\left(\gtrsim 10^{3}\right)$ than there are currently available; such (future) experiments possibly require the use of active grids. ${ }^{5-7}$ Nevertheless, it should be clear that, from observing the trends in Figure 14, to reach the 5/3 scaling range, where the constants are approximately universal, i.e., $C_{\theta}{ }^{+}-C_{u}{ }^{+} \approx C_{\theta}-C_{u}$, the value of $R_{\lambda}$ needs to far exceed $10^{3}$.

\section{DISCUSSION}

According to Figure 11, the secondary contraction plays a dominant role in controlling the scaling-range exponents $\left(m_{u}\right.$ and $\left.m_{\theta}\right)$ since the effect of grid geometry is reduced by the contraction. ${ }^{28,29}$ To gain some understanding of the effects of larger contractions, we reviewed the spectra reported by Uberoi ${ }^{30}$ (Figure 15(b)). To conform with linear theory, Uberoi plotted his velocity spectrum as a function $c k_{1}$, where $c \gg 1$ is the contraction ratio. Uberoi's measurements show that, by increasing $c$, this tends to shift the turbulent energy to the higher wavenumbers and increase the magnitude of the scaling-range exponent, i.e., $m_{u} \propto c$ (see also Figures $8(\mathrm{a})$ and 8(b)). Since $m_{u}$ and $a_{u}$ are related according to $m_{u}=5 / 3-a_{u} R_{\lambda}{ }^{-2 / 3}$ (Eq. (26)), we therefore suspect that, for a fixed Reynolds number $\left(R_{\lambda}<10^{2}\right)$, the magnitude of $a_{u}$ should decrease with increasing contraction ratio, i.e., $a_{u} \propto c^{-1}$.

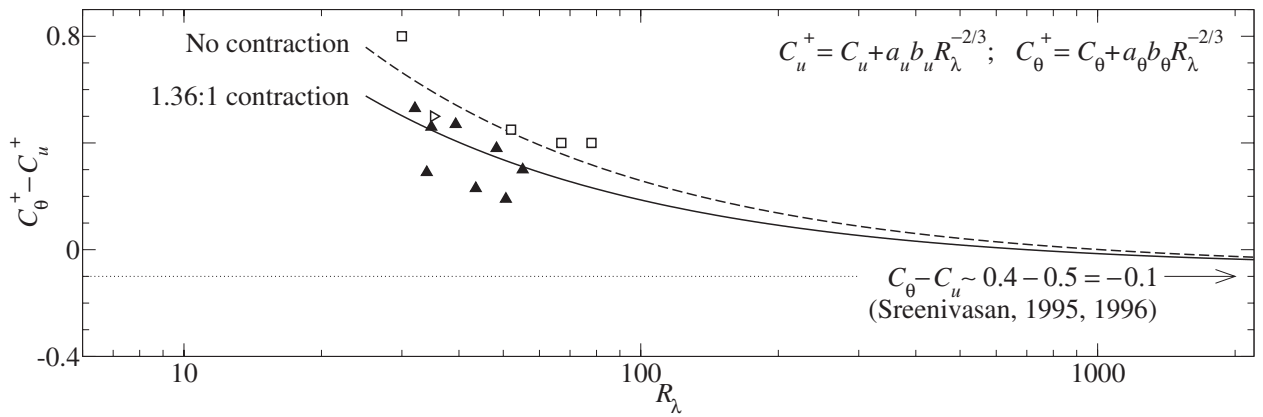

FIG. 14. The difference between the scaling-range coefficients, $C_{\theta}{ }^{+}-C_{u}{ }^{+}$, as a function of $R_{\lambda}$. The present measurements for Rd44w with the contraction are shown as $\boldsymbol{\Lambda}$. With no contraction, the Sq35 measurements are shown as $\square$ and the data point of Yeh and Van Atta ${ }^{52}$ is shown as $\triangleright$. Each curve fit is given by Eq. (33). The asymptotic value $C_{\theta}-C_{u} \sim-0.1$ follows the recommendation of Sreenivasan. ${ }^{17,18}$ 

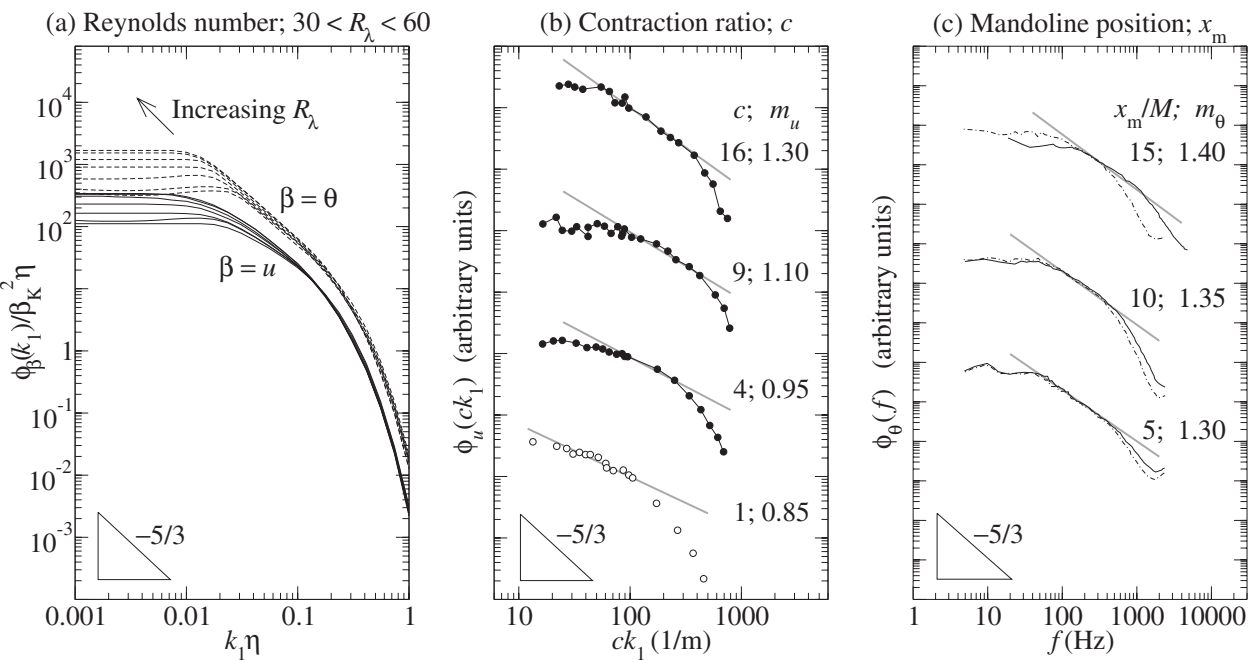

FIG. 15. (a) Present $u$ and $\theta$ measurements for grid Rd44w with contraction (ratio $c=1.36$; location $x_{\mathrm{c}} / M=11$ ) and mandoline (location $x_{\mathrm{m}} / M=1.5$ ) showing the effect of increasing Reynolds number $\left(30<R_{\lambda}<60\right)$ at $t U_{\mathrm{o}} / M \approx 70$. (b) Uberoi's ${ }^{30}$ measurements for $R_{M}=3710$ showing the effect of increasing contraction ratio; the pre-contraction $\left(x_{\mathrm{c}} / M=21\right)$ data are shown as $\bigcirc$; the post-contraction $\left(c=4,9\right.$, and 16) data are shown as $\bullet$; vertical offset is 2 decades. (c) Warhaft's ${ }^{59}$ measurements for grid Sq34 with contraction $\left(c=4 ; x_{\mathrm{c}} / M=60\right)$ at $R_{M}=9700$ showing the effect of increasing streamwise distance $\left(x_{\mathrm{m}} / M=5,10\right.$, and 15) between the mandoline and the turbulence-generating grid; the dotted-dashed lines are for $x / M=53$ (upstream of contraction) and the solid lines (with slope $m_{\theta}$ ) are for $x / M=69$ (downstream of contraction); vertical offset is arbitrary.

From the experimental results summarised in Table II, we find that, while the magnitude of $a_{u}$ may change with different initial conditions (in this case, it is mainly the secondary contraction), the ratio $a_{u} / a_{\theta} \approx 2.0$ is generally unaffected. This finding seems consistent with that of Jayesh $e t$ al. ${ }^{57}$ From their measurements over the Reynolds-number range $30 \lesssim R_{\lambda} \lesssim 130$ using different grids and methods of heating, Jayesh et al. ${ }^{57}$ obtained $m_{u}=1.34$ and $m_{\theta}=1.58$, where the ratio $a_{u} / a_{\theta}=(5 / 3$ $\left.+m_{u}\right) / m_{\theta} \approx 1.9$ is independent of $R_{\lambda}$. Their result is in close agreement with both the present finding for $a_{u} / a_{\theta}$ (Table II) and the proposal of Danaila and Antonia ${ }^{23}$ (Eq. (11)).

The method of heating is of interest as it affects the turbulent mixing. With the "mandoline" technique, the resulting temperature field is sufficiently homogeneous, ${ }^{38,58}$ and the velocity/scalar time scale ratio, which is an important parameter for modelling, ${ }^{13,16}$ tends to be closer to unity than that obtained by heating the flow with the turbulence-generating grid ${ }^{38}$ or with a plenum "toaster." 58 With the mandoline, the initial scale of the thermal fluctuations can be controlled by adjusting the distance $\left(x_{\mathrm{m}}\right)$ between the mandoline and the grid. Figure 15(c) shows that, for a small value of $x_{\mathrm{m}}$ $(\lesssim 10 M)$, the spectra for $\theta$ obtained at both the inlet and exit planes of a 4:1 secondary contraction

TABLE II. Summary of parameters for the curve fits shown in Figures 9(c) and 11-14. The measurements marked "** are the same as those first reported by Mydlarski and Warhaft. ${ }^{5}$ Those marked " $\oplus$ " are in conformity with the proposal of Danaila and Antonia. ${ }^{23}$ The constants marked " $\otimes$ " are selected to minimise the rms difference between the curve fit and the experimental data, and are consistent with the recommendations of Sreenivasan, ${ }^{17,18}$ i.e., $C_{u} \sim 0.5$ and $C_{\theta} \sim 0.4$.

\begin{tabular}{|c|c|c|c|c|c|}
\hline \multirow[b]{2}{*}{ Eq. } & \multirow[b]{2}{*}{ Symbol } & \multicolumn{2}{|c|}{ Contraction ratio } & \multirow[b]{2}{*}{ Description } & \multirow[b]{2}{*}{ Note } \\
\hline & & $\bar{c}=1$ & $c=1.36$ & & \\
\hline (26) & $a_{u}$ & $5.25^{*}$ & 4.25 & The rate-of-change in the scaling-range exponent $m_{u}=\mathrm{f}\left(R_{\lambda}\right)$. & $a_{u} \propto c^{-1} ; c \geq 1$ \\
\hline (27) & $a_{u} / a_{\theta}$ & $2.0^{\oplus}$ & $2.0^{\oplus}$ & The ratio $\left(5 / 3+m_{u}\right) / m_{\theta}$ (the uncertainty is \pm 0.02 ). & $a_{u} / a_{\theta}$ is constant \\
\hline$(28)$ & $b_{u}$ & $2.4^{*}$ & 1.6 & The rate-of-change in the scaling-range coefficients for & $b_{u}<b_{\theta}$ \\
\hline (29) & $b_{\theta}$ & 7.5 & 5.8 & optimally compensated velocity and scalar spectra. & \\
\hline$(28)$ & $C_{u}$ & $0.51^{\otimes}$ & $0.51^{\otimes}$ & The Kolmogorov constant and the Obukhov-Corrsin & $C_{u}$ and $C_{\theta}$ are \\
\hline (29) & $C_{\theta}$ & $0.44^{\otimes}$ & $0.44^{\otimes}$ & constant for a $5 / 3$ power-law scaling range. & constant; $C_{u} \gtrsim C_{\theta}$ \\
\hline
\end{tabular}


are nearly the same. According to Warhaft' ${ }^{59}$ measurements, increasing $x_{\mathrm{m}}$ tends to shift the postcontraction $(\theta)$ spectrum to a higher frequency and slightly increases the magnitude of $m_{\theta}$; this has an overall effect of increasing the time scale ratio ${ }^{59}$ (i.e., $\tau / \tau_{\theta} \approx n_{\theta} / n_{u}$ ) from 1.5 (at $x_{\mathrm{m}}=5 M$ ) to 2.3 (at $x_{\mathrm{m}}=15 M$ ).

The velocity and the temperature scaling-range coefficients $\left(C_{u}{ }^{+}\right.$and $\left.C_{\theta}{ }^{+}\right)$tend towards the Kolmogorov and the Obukhov-Corrsin constants $\left(C_{u}\right.$ and $\left.C_{\theta}\right)$ at different rates. Figure 9(c) shows that the rate-of-change in the scaling-range coefficient, with respect to the scaling-range exponent, is slower for the velocity than for the passive scalar, i.e., $b_{u}<b_{\theta}$. A small reduction in the magnitudes for both $b_{u}$ and $b_{\theta}$ is obtained by using the 1.36:1 contraction (Table II). How $b_{u}$ and $b_{\theta}$ behave for more extreme contractions (i.e., $c \gg 1$ ) is a topic for future investigation.

At low Reynolds numbers $R_{\lambda} \lesssim 10^{2}$, the effect of the 1.36:1 contraction on the scaling-range coefficients $C_{u}{ }^{+}$and $C_{\theta}{ }^{+}$is evident. A comparison of the present data in Figure 13 with the data of Comte-Bellot and Corrsin ${ }^{55}$ shows that, with the present contraction, $C_{u}{ }^{+} \approx 1.0$. With no contraction, the magnitude of the velocity scaling-range coefficient $\left(C_{u}{ }^{+} \approx 1.5\right)$ tends to be larger. Figures 13 and 14 suggest that, as the Reynolds number is increased $\left(R_{\lambda}>10^{2}\right)$, effects due to different initial conditions should diminish and the difference between $\mathrm{C}_{u}{ }^{+}$and $\mathrm{C}_{\theta}{ }^{+}$becomes negligible. From the present curve fits (Table II), the prediction for the asymptotic value $C_{\theta}-C_{u} \sim-0.1$ follows the recommendation of Sreenivasan. ${ }^{17,18}$

In summary, the present findings provided some new insight into how the scaling-range coefficients $\left(\mathrm{C}_{u}{ }^{+}\right.$and $\left.\mathrm{C}_{\theta}{ }^{+}\right)$tend towards the universal constants for closely isotropic turbulence. Clearly, the manner in which $C_{u}{ }^{+}$and $C_{\theta}{ }^{+}$approach universality (i.e., $C_{u}$ and $C_{\theta}$ ) is not unique and that this depends on initial conditions. Indeed, if $R_{\lambda}$ is much less than $10^{2}$, the velocity and scalar fields behave differently, and the initial conditions (e.g., changes to the contraction size and/or the method of heating) will affect the morphology of the two fields. Such information is useful if an attempt is to be made to improve any predictive models for turbulent mixing.

\section{CONCLUSION}

The use of a smooth (1.36:1) contraction improves the isotropy of the large scales for turbulent flows downstream of three different grid geometries. The introduction of a passive scalar by using a mandoline heater located just downstream of the grid allows comparison between the (spectral) characteristics of the velocity and the scalar (temperature) fields with and without the effect of the contraction.

The present grid turbulence closely follows a power-law rate of decay. Although the heating is carefully controlled such that the velocity/scalar time scale ratio is close to 1 , the magnitude of the power-law decay exponent is consistently slightly larger for the scalar than for the velocity (i.e., $n_{\theta} / n_{u} \approx 1.03$ to 1.04 ), independently of the grid geometry.

For low Taylor microscale Reynolds numbers $\left(R_{\lambda} \lesssim 10^{2}\right)$, the thermal spectrum has a more prominent scaling range than the velocity spectrum. The spectral scaling range follows a power law and the scaling-range exponent is larger for the scalar than for the velocity, i.e., $m_{\theta}>m_{u}$. The magnitudes for $m_{u}$ and $m_{\theta}$ are closer to the Kolmogorov value of 5/3 with the 1.36:1 contraction than with no contraction. As the Reynolds number is increased, $m_{\theta}$ approaches $5 / 3$ more rapidly than $m_{u}$, and the difference between $m_{\theta}$ and $m_{u}$ diminishes according to the form $m_{\theta}-m_{u} \propto R_{\lambda}{ }^{-2 / 3}$. The ratio $\left(5 / 3+m_{u}\right) / m_{\theta}(\approx 2.0 \pm 0.02)$ tends to be unaffected by initial conditions and conforms closely with the findings of Danaila and Antonia. ${ }^{23}$

The velocity and temperature scaling-range coefficients of the (optimally) compensated spectra $C_{u}{ }^{+}$and $C_{\theta}{ }^{+}$increase linearly with their respective scaling-range exponents $5 / 3-m_{u}$ and $5 / 3-m_{\theta}$. The magnitudes of the rate-of-change in ${C_{u}}^{+}$and $C_{\theta}{ }^{+}$with respect to their scaling-range exponents are smaller with the 1.36:1 contraction than with no contraction. For the same magnitude of $m_{u}$ and $m_{\theta}$ or for a fixed $R_{\lambda}\left(\lesssim 10^{2}\right)$, the scaling-range coefficient is generally smaller for the velocity spectrum than for the scalar spectrum, i.e., $C_{u}{ }^{+}<C_{\theta}{ }^{+}$. The difference between the scalar and the velocity scaling-range coefficients reasonably follows the form $\left(C_{\theta}{ }^{+}-C_{u}{ }^{+}\right)-\left(C_{\theta}-C_{u}\right) \propto R_{\lambda}{ }^{-2 / 3}$. The scaling-range coefficients $C_{u}{ }^{+}$and $C_{\theta}{ }^{+}$are unlikely to reach $C_{u}$ (the Kolmogorov constant) and $C_{\theta}$ (the Obukhov-Corrsin constant) for the values of $R_{\lambda}$ below $10^{3}$. The manner in which $C_{u}$ and $C_{\theta}$ 
are approached (from small $R_{\lambda}$ ) should be useful for the purpose of refining theory/model to predict turbulent mixing when the effect of finite Reynolds number cannot be neglected.

On a more general note, the present results suggest that the use of the contraction which improves the isotropy at the large scales leads to the parameters such as $m_{u}$ and $m_{\theta}$ (Figure 11) being closer to the expected asymptotic values corresponding to very large $R_{\lambda}$. This in turn implies that it would be worthwhile to try and extend the present range of $R_{\lambda}$ before reappraising the question, addressed, for example, by Antonia and Burattini ${ }^{19}$ based on imperfectly isotropic turbulence data, of how large should $R_{\lambda}$ be in order to attain the " $4 / 5$ " law of Kolmogorov.

\section{ACKNOWLEDGMENTS}

The Australian Research Council and the Natural Sciences and Engineering Research Council of Canada are gratefully acknowledged for their financial support.

${ }^{1}$ A. N. Kolmogorov, "The local structure of turbulence in incompressible viscous fluid for very large Reynolds numbers," Dokl. Akad. Nauk SSSR 30, 299 (1941) [Proc. R. Soc. London, Ser. A 434, 9-13 (1991)].

${ }^{2}$ A. N. Kolmogorov, "Dissipation of energy in the locally isotropic turbulence," Dokl. Akad. Nauk SSSR 32, 16 (1941) [Proc. R. Soc. London, Ser. A 434, 15 (1991)].

${ }^{3}$ A. M. Obukhov, "Structure of the temperature field in turbulent flow," Izv. Akad. Nauk SSSR, Ser. Geogr. Geofiz. 13, 58 (1949).

${ }^{4}$ S. Corrsin, "On the spectrum of isotropic temperature fluctuations in an isotropic turbulence," J. Appl. Phys. 22, 469 (1951).

${ }^{5}$ L. Mydlarski and Z. Warhaft, “On the onset of high-Reynolds-number grid-generated wind tunnel turbulence,” J. Fluid Mech. 320, 331 (1996)

${ }^{6}$ L. Mydlarski and Z. Warhaft, "Passive scalar statistics in high-Péclet-number grid turbulence," J. Fluid Mech. 358, 135 (1998).

${ }^{7}$ H. S. Kang, S. Chester, and C. Meneveau, "Decaying turbulence in an active-grid-generated flow and comparisons with large-eddy simulation," J. Fluid Mech. 480, 129 (2003).

${ }^{8}$ Z. Warhaft, "Why we need experiments at high Reynolds numbers," Fluid Dyn. Res. 41, 021401 (2009).

${ }^{9}$ P. K. Yeung and Y. Zhou, "Universality of the Kolmogorov constant in numerical simulations of turbulence," Phys. Rev. E 56, 1746 (1997).

${ }^{10}$ T. Gotoh, D. Fukayama, and T. Nakano, "Velocity field statistics in homogeneous steady turbulence obtained using a high-resolution direct numerical simulation," Phys. Fluids 14, 1065 (2002).

${ }^{11}$ P. A. Durbin, "A stochastic model of two-particle dispersion and concentration fluctuations in homogeneous turbulence," J. Fluid Mech. 100, 279 (1980).

${ }^{12}$ P. A. Durbin, "Analysis of the decay of temperature fluctuations in isotropic turbulence," Phys. Fluids 25, 1328 (1982).

${ }^{13}$ B. L. Sawford, "Micro-mixing modelling of scalar fluctuations for plumes in homogeneous turbulence," Flow, Turbul. Combust. 72, 133 (2004).

${ }^{14}$ T. Watanabe and T. Gotoh, "Statistics of a passive scalar in homogeneous turbulence," New J. Phys. 6, 40 (2004).

${ }^{15}$ P. K. Yeung, D. A. Donzis, and K. R. Sreenivasan, "High-Reynolds-number simulation of turbulent mixing," Phys. Fluids 17, 081703 (2005).

${ }^{16}$ S. Viswanathan and S. B. Pope, "Turbulent dispersion from line sources in grid turbulence," Phys. Fluids 20, 101514 (2008).

${ }^{17}$ K. R. Sreenivasan, "On the universality of the Kolmogorov constant," Phys. Fluids 7, 2778 (1995).

${ }^{18}$ K. R. Sreenivasan, "The passive scalar spectrum and the Obukhov-Corrsin constant," Phys. Fluids 8, 189 (1996).

${ }^{19}$ R. A. Antonia and P. Burattini, "Approach to the 4/5 law in homogeneous isotropic turbulence," J. Fluid Mech. 550, 175 (2006).

${ }^{20}$ L. Danaila, R. A. Antonia, and P. Burattini, "Comparison between kinetic energy and passive scalar energy transfer in locally homogeneous isotropic turbulence," Phys. D 241, 224 (2012).

${ }^{21}$ A. M. Yaglom, "On the local structure of a temperature field in a turbulent flow," Dokl. Akad. Nauk SSSR 69, 743 (1949).

${ }^{22}$ K. R. Sreenivasan, "On local isotropy of passive scalars in turbulent shear flows," Proc. R. Soc. London, Ser. A 434, 165 (1991).

${ }^{23}$ L. Danaila and R. A. Antonia, "Spectrum of a passive scalar in moderate Reynolds number homogeneous isotropic turbulence," Phys. Fluids 21, 111702 (2009).

${ }^{24}$ G. K. Batchelor, "Small-scale variation of convected quantities like temperature in turbulent fluid.Part 1. General discussion and the case of small conductivity," J. Fluid Mech. 5, 113 (1959).

${ }^{25}$ R. H. Kraichnan, "Inertial-range transfer in two- and three-dimensional turbulence," J. Fluid Mech. 47, 525 (1971).

${ }^{26}$ R. A. Antonia, T. Zhou, and Y. Zhu, "Three-component vorticity measurements in a turbulent grid flow," J. Fluid Mech. 374, 29 (1998)

${ }^{27}$ G. Comte-Bellot and S. Corrsin, "The use of a contraction to improve the isotropy of grid-generated turbulence," J. Fluid Mech. 25, 657 (1966).

${ }^{28}$ P. Lavoie, "Effects of initial conditions on decaying grid turbulence," Ph.D. dissertation (University of Newcastle, Newcastle, Australia, 2006). 
${ }^{29}$ P. Lavoie, L. Djenidi, and R. A. Antonia, "Effects of initial conditions in decaying turbulence generated by passive grids," J. Fluid Mech. 585, 395 (2007).

${ }^{30}$ M. S. Uberoi, "Effect of wind-tunnel contraction on free-stream turbulence," J. Aeronaut. Sci. 23, 754 (1956).

${ }^{31}$ R. A. Antonia, P. Lavoie, L. Djenidi, and A. Benaissa, "Effect of a small axisymmetric contraction on grid turbulence," Exp. Fluids 49, 3 (2010).

${ }^{32}$ R. R. Mills and S. Corrsin, "Effect of contraction on turbulence and temperature fluctuations generated by a warm grid," Natl. Aeronaut. Space Adm. Memo. 5-5-59W (1959).

${ }^{33}$ S. K. Lee, A. Benaissa, L. Djenidi, P. Lavoie, and R. A. Antonia, "Decay of passive-scalar fluctuations in slightly stretched grid turbulence," Exp. Fluids (to be published).

${ }^{34}$ G. K. Batchelor, The Theory of Homogeneous Turbulence (Cambridge University Press, Cambridge, 1953).

${ }^{35}$ T. Zhou, R. A. Antonia, L. Danaila, and F. Anselmet, "Transport equations for the mean energy and temperature dissipation rates in grid turbulence," Exp. Fluids 28, 143 (2000).

${ }^{36}$ T. Zhou, R. A. Antonia, and L. P. Chua, "Performance of a probe for measuring turbulent energy and temperature dissipation rates," Exp. Fluids 33, 334 (2002).

${ }^{37}$ R. A. Antonia, R. J. Smalley, T. Zhou, F. Anselmet, and L. Danaila, "Similarity solution of temperature structure functions in decaying homogeneous isotropic turbulence," Phys. Rev. E 69, 016305 (2004).

${ }^{38}$ Z. Warhaft and J. L. Lumley, "An experimental study of the decay of temperature fluctuations in grid-generated turbulence," J. Fluid Mech. 88, 659 (1978).

${ }^{39}$ S. K. Lele, "Compressibility effects on turbulence," Annu. Rev. Fluid Mech. 26, 211 (1994).

40 J. C. Wyngaard, "Measurement of small-scale turbulence structure with hot wires," J. Sci. Instrum. 1, 1105 (1968).

${ }^{41}$ L. W. B. Browne and R. A. Antonia, "The effect of wire length on temperature statistics in a turbulent wake," Exp. Fluids 5, 426 (1987).

${ }^{42}$ R. J. Moffat, "Describing the uncertainties in experimental results," Exp. Therm. Fluid Sci. 1, 3 (1988).

${ }^{43}$ P. D. Welch, "The use of fast Fourier transform for the estimation of power spectra: A method based on time averaging over short, modified periodograms," IEEE Trans. Audio Electroacoust. AU-15, 70 (1967).

${ }^{44}$ R. A. Antonia, L. W. B. Browne, and A. J. Chambers, "Determination of time constants of cold wires," Rev. Sci. Instrum. 52, 1382 (1981).

${ }^{45}$ L. Djenidi and R. A. Antonia, "A spectral chart method for estimating the mean turbulent kinetic energy dissipation rate," Exp. Fluids (to be published).

${ }^{46}$ S. B. Pope, Turbulent Flows (Cambridge University Press, Cambridge, 2000).

${ }^{47}$ H. Abe, R. A. Antonia, and H. Kawamura, "Correlation between small-scale velocity and scalar fluctuations in a turbulent channel flow," J. Fluid Mech. 627, 1 (2009).

${ }^{48}$ R. A. Antonia and H. Abe, "Inertial range similarity for velocity and scalar spectra in a turbulent channel flow," in Proceedings of the 6th Turbulence Heat Mass Transfer, edited by K. Hanjalić, Y. Nagano, and S. Jakirlić (Begell House, Inc., 2009), p. 119.

${ }^{49}$ R. A. Antonia and P. Orlandi, "Effect of Schmidt number on small-scale passive scalar turbulence," Appl. Mech. Rev. 56, $615(2003)$

${ }^{50}$ P. Sepri, "Two-point turbulence measurements downstream of a heated grid," Phys. Fluids 19, 1876 (1976).

${ }^{51}$ C. Rey and A. Boudjemaa, "Free convection grid turbulence," in Proceedings of the 2nd European Turbulence Conference (Adv. Turb. 2), edited by H. H. Fernholz and H. E. Fiedler (Springer-Verlag, 1989), p. 204.

${ }^{52}$ T. T. Yeh and C. W. Van Atta, "Spectral transfer of scalar and velocity fields in heated-grid turbulence," J. Fluid Mech. 58, 233 (1973).

${ }^{53}$ J. Schedvin, G. R. Stegen, and C. H. Gibson, “Universal similarity at high grid Reynolds numbers,” J. Fluid Mech. 66, 561 (1974).

${ }^{54}$ A. L. Kistler and T. Vrebalovich, "Grid turbulence at large Reynolds numbers," J. Fluid Mech. 26, 37 (1966).

${ }^{55}$ G. Comte-Bellot and S. Corrsin, "Simple Eulerian time correlation of full- and narrow-band velocity signals in gridgenerated, 'isotropic' turbulence," J. Fluid Mech. 48, 273 (1971).

${ }^{56}$ S. G. Saddoughi and S. V. Veeravalli, "Local isotropy in turbulent boundary layers at high Reynolds number," J. Fluid Mech. 268, 333 (1994).

${ }^{57}$ Jayesh, C. Tong, and Z. Warhaft, "On temperature spectra in grid turbulence," Phys. Fluids 6, 306 (1994).

${ }^{58}$ A. Sirivat and Z. Warhaft, "The effect of a passive cross-stream temperature gradient on the evolution of temperature variance and heat flux in grid turbulence," J. Fluid Mech. 128, 323 (1983).

${ }^{59} \mathrm{Z}$. Warhaft, "An experimental study of the effect of uniform strain on thermal fluctuations in grid-generated turbulence," J. Fluid Mech. 99, 545 (1980). 